\title{
Edge Computing und Industrie 4.0
}

\section{Anwendungsbereiche in der Schweizer Fertigungsindustrie}

\author{
Dominik Appius, Roger Andreas Probst und Kim Oliver Tokarski
}

\section{Zusammenfassung}

Durch die industrielle, digitale Transformation, insbesondere durch die Vernetzung von Fertigungsanlagen, wird zusehends eine sehr große Datenmenge in der Schweizer Fertigungsindustrie generiert. Viele Daten bleiben dabei lokal (oft) ungenutzt oder werden über weite Transportwege an zentrale Rechenzentren zur Analyse gesendet. Vor diesem Hintergrund stellt sich die Frage, wie Daten so genutzt werden können, dass lange Transportwege entfallen und zeitgleich, durch die Verarbeitung dieser Daten, Wissen generiert werden kann. Dieser Beitrag liefert erste Antworten auf der Basis von empirischen Erkenntnissen, welche durch Befragungen von Anbietern, Beratungsunternehmen und Fertigungsunternehmen im Bereich Edge Computing durchgeführt wurden. Dabei liefert die vorliegende Studie Erkenntnisse in den Bereichen technisches Verständnis, Geschäftsmodelle und Anwendungsszenarien sowie praktische Umsetzungen im Sinne von Pilotierungen und Rollouts als Proof of Concept.

\subsection{Einleitung}

In den Wirtschaftswissenschaften werden „klassischerweise“ Arbeit, Boden und Kapital als die 3 wichtigsten Ressourcen zur Güterproduktion betrachtet (Neubäumer et al. 2017). Ergänzend und vielleicht teilweise ersetzend werden Daten und deren Nutzung als ,neue

D. Appius $(\bowtie) \cdot$ R. A. Probst

Bern, Schweiz

K. O. Tokarski

Berner Fachhochschule Wirtschaft, Bern, Schweiz

E-Mail: kim.tokarski@bfh.ch 
Ressource" durch die digitale Transformation von Wirtschaft und Gesellschaft zunehmend bedeutender. Sie gelten für die Wirtschaft als ,Schlüsselressource“ für den unternehmerischen Erfolg (Seeberg 2016). Beispielhaft wird das Wachstum des globalen Datenvolumens von 33 Zettabyte im Jahr 2018 auf rund 175 Zettabyte im Jahr 2025 prognostiziert (Reinsel et al. 2018; Gombos 2019). Dieses starke, exponentielle Wachstum bringt klassische IT-Architekturmodelle an Tragfähigkeitsgrenzen und bedarf neuer, innovativer Konzepte im Umgang mit Daten und deren Verarbeitung (IT Verlag für Informationstechnik GmbH 2017). Bis dato wurden fehlende Rechenkapazitäten meist mit dem Ausbau bestehender zentraler Rechenzentren kompensiert (Mutschler 2017). In vielen Fällen können klassische Rechenzentren aufgrund geografischer Distanzen die Echtzeitanforderungen (beispielsweise Datenübertragung und -verarbeitung) nicht gewährleisten. Jedoch entwickelt sich durch die Industrie 4.0 ein zusätzliches Bedürfnis, nebst der Verarbeitung und dem Speichern von Daten, diese zudem auch in Echtzeit verfügbar und nutzbar zu haben. Durch gezielte Analysen und Verwendungen von Daten entstehen für die Wirtschaft attraktive, neue Geschäftsmodelle entlang der gesamten Wertschöpfungskette. Dies wird umso bedeutender, wenn auf weitere Wachstumstreiber im Kontext von Daten abgestellt wird. Beispielsweise werden bis zum Jahr 2022 schätzungsweise bis zu 50 Mrd. Geräte und Sensoren mit dem Internet verknüpft (Steinhaus 2018). Ein gewichtiger Anteil stammt dabei aus der Fertigungsindustrie. Ausgehend vom Jahr 2017, entspricht dies einem Zuwachs von über $595 \%$ in 5 Jahren (Jansen 2017). Folglich gewinnen die zeitnahe, lokale Datenverarbeitung am Netzwerkrand und somit auch das Konzept des Edge Computing (EC) an Relevanz.

Aufgrund der Kundenerwartungen, der Intensivierung des globalen Wettbewerbs, dem fehlenden Wirtschaftswachstum hiesiger Länder und den steigenden (Rohstoff-)Preisen sind Unternehmen der Schweizer Fertigungsindustrie gefordert, neue, lukrative Geschäftsmodelle, wie auch die digitale Transformation von Entwicklung und Produktion, voranzutreiben (Brüning et al. 2017; Friedli et al. 2018; Hoffmann 2015; Stiehler und Bieber 2016; Grosser 2018). Sich dabei die ,richtigen Gedanken“ zu machen (Effektivität), ausreichend Zeit, Budget und Know-how bereitzustellen und zeitgleich das „Mindset“ der Mitarbeitenden auf diesen Wandel einzustellen, ist eine wesentliche Herausforderung (Brüning et al. 2017; Grosser 2018). Rund zwei Drittel der befragten Führungskräfte einer Fujitsu-Studie stimmen zu, dass es sich hierbei um die bisher größte Herausforderung handelt (Fujitsu Technology Solutions AG 2017).

Mit dem Wandel der digitalen Transformation sind neue Geschäftsmodelle und Technologien verbunden. Dem „Internet of Things“ (IoT) kommt in diesem Kontext eine besondere Bedeutung zu. Das IoT integriert und vernetzt reale Objekte mit dem Internet (Flörkemeier und Mattern 2010). Damit IoT durch die Wirtschaft gewinnbringend eingesetzt werden kann, werden leistungsstarke Modelle wie Edge Computing vorausgesetzt, welche am Rande des Netzwerks in Echtzeit und unter geringen Latenzzeiten die Daten an der Quelle (Maschinendaten, Produktionsdaten etc.) verarbeiten (Ostler 2018). Edge Computing bietet, im Vergleich zu regulären Servern, den Vorteil, vorgelagert an der Datenentstehungsquelle performant und in adaptiven Architekturkonzepten, Daten für die 
weitere Nutzung zu erfassen, auszuwerten und zu versenden. Weiter können diese auch lokal in der Unternehmensumgebung eine profitable Optimierung der Prozesse ermöglichen und ,reguläre“ Server bzw. IT-Infrastrukturen entlasten. Eine zielorientierte Anwendung kann zur Steigerung der Wettbewerbsfähigkeit beitragen und nachhaltige Wettbewerbsvorteile generieren.

Der Entwicklungsstand von Edge Computing kann in Anlehnung an den „Hype Cycle“ für aufkommende Technologien von Gartner gemäß Abb. 13.1 verdeutlicht werden. Edge Computing wurde darin im Jahr 2017 als IT-Designansatz definiert. Dabei wird das Produktivitätsplateau (Phase 5 des Modells) für eine marktfähige Anwendung (bei weitem) noch nicht erreicht. Edge Computing befindet sich in der Einschätzung von Gartner in der 1. Phase, „Innovation Trigger“, und steht dabei knapp an der Grenze zur 2. Phase, „Peak of Inflated Expectations“ (Panetta 2017). Der Einordnung entsprechend sind Herausforderungen, Risiken und Chancen in der Praxis nur wenig bekannt (Ziegler 2017). An dieser Stelle sei angemerkt, dass Edge Computing in den Folgejahren auf Gartners Hype Cycle nur bedingt ausgewiesen wird. Bei den aktuellen Versionen wird hier der Begriff „Edge Analytics" angeführt.

Eingebettet in diesen Hintergrund beschäftigt sich auch die Schweizer Fertigungsindustrie zusehends mit der digitalen Transformation ihrer Prozesse und Anlagen. Dadurch rückt die effizientere, bessere Datenverarbeitung und somit auch Edge Computing in den Fokus der Betrachtungen und Bemühungen (Rudolph 2019; Harting et al. 2015; EUROFORUM Schweiz AG 2019). Aktuell setzen Unternehmen den Designansatz von EC le-

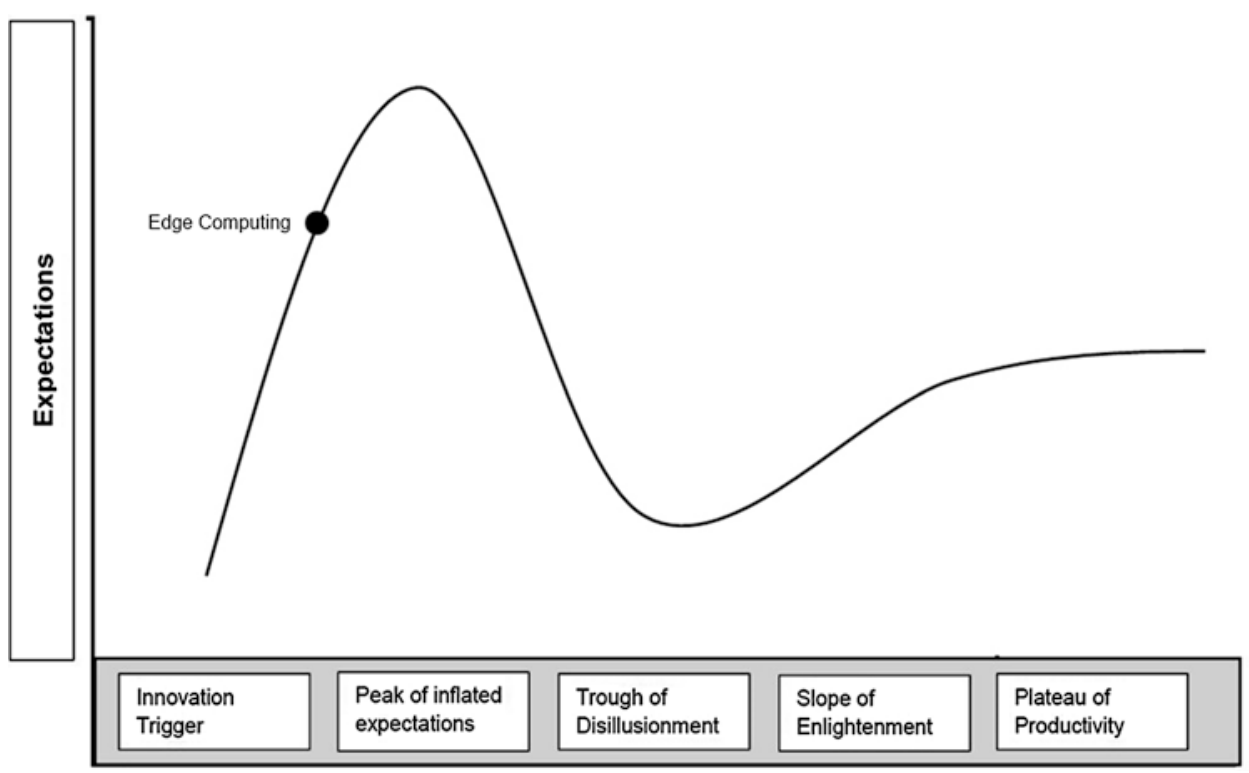

Abb. 13.1 Gartners Hype-Cycle-Modell für aufkommende Technologien mit Fokus auf Edge Computing. (Quelle: eigene Darstellung auf Basis von Stark 2017 und Panetta 2017). 
diglich ergänzend ein oder zögern gar mit der Implementierung (UTK Media GmbH 2018; itelligence AG 2018; IFS Deutschland GmbH und Co. KG/IFS Schweiz AG 2019). Oftmals wird die erforderliche Gewichtung der Thematik vernachlässigt. Dies führt dazu, dass ein kompaktes Grundverständnis, wie auch Analysen und Prognosen von EC für die Schweizer Fertigungsindustrie von besonderem Interesse sind (Grosser 2018; Harting et al. 2015).

\subsection{Theoretische Grundlagen}

Edge Computing ist ein IT-Designansatz, welcher zur Realisierung des IoT eingesetzt werden kann. Dabei wird beim Architekturkonzept von etablierten Netzwerkkomponenten Gebrauch gemacht und keine neuartigen Technologien in den Designansatz involviert (1\&1 IONOS SE 2018; Obiltschnig, Edge und Fog Computing Architekturen im Industrial IoT 2019a). Unter EC wird somit ein Datenverarbeitungsmodell verstanden, welches durch die Bereitstellung von dezentralen IT-Ressourcen generierte Daten dezentral am Entstehungsort, somit am Rande der Netzwerkperipherie, direkt analysiert und weiterverarbeitet (Hewlett Packard Enterprise 2019; Martins und Kobylinska 2019; Sasson 2019). Weiter kann EC als Komplementärlösung und Schlüsselkonzept zu zentralen Konzepten wie der Cloud eingesetzt werden, sodass zukünftige Konzepte im Bereich IoT realisiert werden können (Augmented Reality, Condition Monitoring, Predictive und Preventive Maintenance, Machine Learning etc.) (QSC AG 2017; Goertz und Salge 2019; Martins und Kobylinska 2019). Dabei können 3 etablierte Ausprägungen des EC-Designansatzes angeführt werden: (1) Fog Computing, (2) Cloudlet Computing und (3) Mobile Edge Computing. Dabei wird Fog Computing oft als autonomer IT-Designansatz betrachtet (Borcoci und Obreja 2018; Liu et al. 2018; Mouradin et al. 2017; Dolui und Kanti Datta 2017).

Im Jahr 2020 sollen durch IoT rund 20-30 Mrd. physische Objekte mit dem Internet vernetzt sein (Hüning 2018; Hoti 2019; Zimmermann 2016). Edge Computing ist somit zum Schlüsselkonzept für das IoT geworden, um bereits an der Quelle eine Datenverarbeitung zur nachhaltigen Entlastung, wie etwa der Cloud, durchzuführen (Najmul et al. 2018). Um die Anwendung von EC im Gesamtkontext des IoT aufzuzeigen, werden im Folgenden etablierte IoT-Layer und deren Zusammenspiel mit der Cloud in Abb. 13.2 illustriert.

\section{Layer 1 und 2}

Der 1. Architektur-Layer befasst sich mit den physischen Objekten der realen Welt. Sie werden mittels Sensoren und Aktoren in ein Netzwerk integriert. Dadurch findet eine Transformation zu Smart Objects statt und eine Interaktion zwischen den Elementen wird ermöglicht (Eppenberger Digital 2019). Der Mensch benutzt Sinnesorgane und das Gehirn, um Parameter aus der Umgebung zu erfassen und zu verarbeiten. Genauso übernehmen Sensoren die Messung von physikalischen und technischen Variablen (Druck, Tem- 
peratur, Status, Zustand etc.). Aktoren fungieren als Gegenstück und führen die Aktionen (Maschinen-/Anlagensteuerung, Warnleuchten, Meldungen etc.) auf Basis der gesammelten Daten aus (Hüning 2018). Diese erfolgreiche Interaktion zwischen Sensoren und Aktoren stellt eine Grundvoraussetzung für die Realisierung von IoT-Anwendungen dar (Kahl 2017; Kithion 2016). Sensoren benötigen in der Funktion als Datensammler nebst einer Hardware auch Computing-Ressourcen in Form von Mikroprozessoren, Kommunikationstechniken und Software-Komponenten (Obiltschnig, Was steckt hinter dem Internet der Dinge?). Die Tab. 13.1 illustriert häufig verwendete, technische Eigenschaften von Smart Objects.

\section{Layer 3}

Auf der 3. Ebene wird der Edge Layer eingeordnet. Es werden IT-Ressourcen zwischen den zentralen Rechenzentren und Smart Objects in Form von Edge Devices eingesetzt. Diese

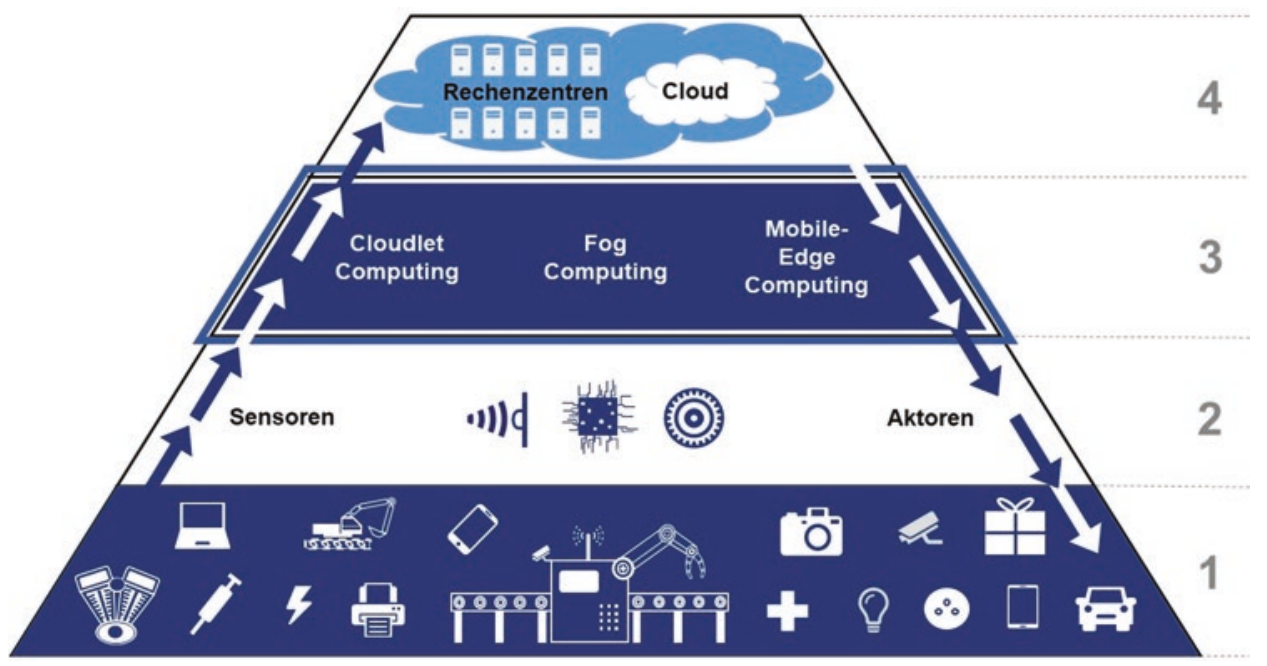

Abb. 13.2 Illustration des Zusammenspiels der Layer. (Quelle: eigene Darstellung)

Tab. 13.1 Häufige Eigenschaften von Smart Objects

\begin{tabular}{l|l|l}
\hline Identifikation & Adressierung & Transporttechnologie \\
\hline - MAC-Adressen & - IPv6 & - 2G GSM, 3G UMTS, 4G, 5G \\
- URI/URL & - IPv4 & - LPWAN \\
- Serialnummern & - 6LoWPAN & - WLAN \\
- IP-Adresse & & - Bluetooth \\
- RFID-Tags & & - ZigBee \\
& & - NFC \\
& & - Ethernet \\
\hline
\end{tabular}

Quelle: Fuqaha et al. (2015), Lee und Crespi (2011), Jung (2017), van Moorsel (2016), Gaggi et al. (2017), Obiltschnig, Was steckt hinter dem Internet der Dinge? 
Edge Devices verwerten mittels IT-Ressourcen die Daten vorzeitig und dezentral, um so den Transport zu zentralen Rechenzentren (oftmals) zu vermeiden, zu reduzieren oder Datenverarbeitungen zu beschleunigen. Als Edge Device können diverse Netzwerkkomponenten eingesetzt werden. Häufig sind dies Edge Router, Edge Gateways oder Edge Server (DATACOM Buchverlag GmbH 2017; Abu Sharkh et al. 2019). Edge Computing kennt unterschiedliche Designansätze, nachfolgend werden die 3 verbreitetsten Standards erläutert (Borcoci und Obreja 2018; Dolui und Kanti Datta 2017; Liu et al. 2018; Mouradin et al. 2017).

\section{Fog Computing}

Das Konzept von Fog Computing wurde vom Telco-Unternehmen Cisco im Jahr 2012 veröffentlicht (Mouradin et al. 2017). Auf dem Edge Layer werden sogenannte Fog Nodes als dezentrale Computing-Infrastruktur benötigt, um die erzeugten Daten von Smart Objects vor dem Transport in die Cloud zu verarbeiten (Dolui und Kanti Datta 2017). Dieses Konzept hat den Vorteil, dass im Gengensatz zu den 2 nachfolgenden Konzepten durch die unterschiedliche Positionierung von IT-Ressourcen mehr Flexibilität in der EdgeAnwendung ermöglicht wird (Dolui und Kanti Datta 2017; Borcoci und Obreja 2018). Durch die Flexibilität variiert die Anzahl der Fog Nodes (Router, Switches, Access Points, Gateways, Computer etc.) oft, sodass die Anwendung von Fog Computing in vielen Fällen äußerst heterogen und vielschichtig ist (Borcoci und Obreja 2018; Dolui und Kanti Datta 2017; Mouradin et al. 2017). Fog Computing kann jedoch, gesamtheitlich betrachtet nicht, wie das Mobile Edge Computing oder das Cloudlet Computing, Stand-Alone betrieben werden. Es benötigt daher eine vorhandene Cloud-Infrastruktur zur Implementierung (Mouradin et al. 2017).

\section{Cloudlet-Computing}

Das Konzept des Cloudlet-Computing wurde bereits im Jahr 2009 durch den Wissenschaftler Satyanarayanan veröffentlicht und wird oft als Datacenter-in-a-Box beschrieben, welches unter anderem für Augmented Reality (AR) Anwendung findet (Mouradin et al. 2017; Rüdiger und Ostler 2019). Der Ansatz sieht ein Mikro-Datacenter aus einem Server oder Cluster von Servern zwischen der Cloud und den Smart Objects vor und bedient sich auch technologischen Standards der Cloud (Borcoci und Obreja 2018; Mouradin et al. 2017). Einzelne Server oder Server-Cluster stellen die notwendige Rechenleistung und Speichersysteme zur Verfügung und werden oft auch als Cloud in der Edge bezeichnet (Dolui und Kanti Datta 2017; Mouradin et al. 2017). Das Cloudlet Computing ist auf ressourcenintensive Mobile Devices ausgerichtet und stellt für das Mobile Offloading die notwendigen IT-Ressourcen bereit (Borcoci und Obreja 2018; Dolui und Kanti Datta 2017; Mouradin et al. 2017). Als vorteilhafte Eigenschaft zu Fog Computing ist hervorzuheben, dass mehr Rechenleistung und Bandbreite zur Verfügung gestellt werden kann, was die Echtzeitanforderungen von Mobile-Applikationen besser erfüllt. Da Cloudlet über die Kommunikationstechnologie WiFi funktioniert, ist der Ansatz äußerst energieschonend, schränkt aber zugleich die Flexibilität der Anwendung ein (Borcoci und Obreja 2018; Dolui und Kanti Datta 2017; Rüdiger und Ostler 2019; Volk 2014). 


\section{Mobile Edge Computing}

Der Ansatz des Mobile Edge Computing (MEC) wurde vom European Telecommunication Standard Institute konzipiert und 2014 veröffentlicht. Der Ansatz wird vom Industriekonsortium getrieben und soll beispielsweise Projekte im Bereich Augmented Reality und IoT realisieren (Mouradin et al. 2017; Liu et al. 2018; Borcoci und Obreja 2018). Der Ansatz sieht MEC-Server als Edge-Device/s am Rande des Netzwerkes vor, welche nahe zu den Basisstationen positioniert werden und somit IT-Ressourcen bereitstellen. Diese Stationen betreiben ein Radio Access Netzwerk (RAN), welches entgegen der Cloudlet nebst mobilen Devices auch nichtmobilen Devices eine Verbindung im Netzwerk ermöglicht (Liu et al. 2018; Borcoci und Obreja 2018; Dolui und Kanti Datta 2017; Mouradin et al. 2017; Taleb et al. 2017). Der Fokus liegt in erster Linie auf den Echtzeitanforderungen durch Latenzreduzierung, damit die hohen Bandbreiten einen schnellen Datentransport zwischen den Endgeräten und dem MEC-Server ermöglichen (Borcoci und Obreja 2018; Dolui und Kanti Datta 2017; Liu et al. 2018). Da der Ansatz auf Mobilnetzwerken basiert, verspricht der Ansatz eine erhebliche Verbesserung der Latenz durch die Einführung von 5G (Borcoci und Obreja 2018; Mouradin et al. 2017).

\section{Layer 4}

Die Cloud ist eine ,allgegenwärtige“ und „günstige“ IT-Infrastruktur, welche über das Internet ortsunabhängig IT-Ressourcen in Form von Servern, Speichersystemen, Applikationen und Services zur Verfügung stellt (Mell und Grance 2011; Stergiou et al. 2016). Da das generierte Datenvolumen kontinuierlich zunimmt, stößt die Cloud (teilweise) an ihre Tragfähigkeitsgrenzen. Kombiniert mit der geografischen Distanz entstehen höhere Latenzzeiten und eine Bandbreitenüberlast (El-Sayed et al. 2018). Dies führt beispielweise, durch „späte bzw. verspätete“ Reaktion in der Produktionsstraße, zu Ausschuss, zu Maschinenausfällen oder Fabrikationsverzögerungen. Edge Computing optimiert die Datenweitergabe in Richtung Cloud und behebt die genannten Probleme, um so beispielsweise den Weg für die Anwendung im Bereich IoT zu gewährleisten (Gross 2018).

In der Gesamtbetrachtung der Layer 1-4 kann somit festgehalten werden, dass EC kein isolierter Designansatz ist. Vielmehr bettet er sich in das IoT ein und funktioniert in Kombination mit anderen Konzepten, wie beispielsweise Big Data, aber auch künstlicher Intelligenz. Durch die globale, digitale Transformation entstehen dabei Herausforderungen, die es zu bewältigen gilt. Dabei spielt der Einsatz von EC eine wichtige Rolle und bietet unterschiedliche Nutzenmöglichkeiten, die in Tab. 13.2 in ausgewählter Form erläutert werden.

Edge Computing kennt in der Industrie 4.0 diverse Anwendungsgebiete. Nachfolgend werden einige zur Verständnisförderung erläutert. Meistgenannt wird der Einsatz von EC im Zusammenhang mit AR bzw. Cognitive Assistance Applications. Dabei unterstützen spezielle Brillen (Head Mounted Displays - HMD), wie z. B. die Microsoft HoloLens, die Mitarbeitenden durch visuelle Anleitungen auf dem Display und durch Audioausgaben im Herstellungs- und Qualitätssicherungsprozess. Edge Computing bietet hierbei geringere Latenzzeiten als das Cloud Computing. Besonders Fog Computing eignet sich gemäß dem Projekt Shipyard 4.0 von Navantia beim Transport vieler kleinerer Datenmengen via WiFi. 
Tab. 13.2 Nutzenableitung von Edge Computing

\begin{tabular}{|c|c|c|}
\hline & Herausforderungen der Digitalisierung & Nutzen Edge Computing (EC) \\
\hline Datensphäre & $\begin{array}{l}\text { - Ungefähres Wachstum der Datensphäre } \\
\text { durch IoT: } \\
\text { - 2018: } 40 \text { ZB } \rightarrow \text { 2025: } 175 \text { ZB } \\
\text { - Ø Wachstum von } 438 \% \\
\text { - Ungefährer Zuwachs an Smart Objects/ } \\
\text { IoT Geräten: } \\
\text { - 2018: 2-5 ZB } \rightarrow \text { 2025: } 45 \text { ZB } \\
\text { - Ø Zuwachs von 900-2250 \% }\end{array}$ & $\begin{array}{l}\text { - Eliminierung geografischer } \\
\text { Distanzen für den Datenverkehr } \\
\text { bezweckt: } \\
\text { - Entlastung der Bandbreiten } \\
\text { - Minimierung der Latenz } \\
\text { - Echtzeitanforderungen werden } \\
\text { besser erfüllt und zunehmend } \\
\text { wichtiger } \\
\text { - Entlastung zentraler } \\
\text { Rechenzentren }\end{array}$ \\
\hline Energie & $\begin{array}{l}\text { - Zunehmender Energieverbrauch } \\
\text { - Ausgelöst durch die steigende } \\
\text { Vernetzung und damit verbundene } \\
\text { „energiehungrige“ Anwendungen } \\
\text { - Wireless-Modul braucht Energie für } \\
\text { den Transport } \\
\text { - Zentrale Rechenzentren verwenden } \\
\text { aufgrund der Vernetzung mehr } \\
\text { Energie (3-mal so viel in den } \\
\text { nächsten 10 Jahren) }\end{array}$ & $\begin{array}{l}\text { - Verteilte Infrastrukturen wie EC } \\
\text { benötigen 14-25\% weniger } \\
\text { Energie: } \\
\text { - Minimierung der Überlast } \\
\text { zentraler Rechenzentren } \\
\text { - Verzicht auf größere Kühlsysteme } \\
\text { - Verbesserung von Image/Ansehen } \\
\text { - Kosteneinsparungen trotz } \\
\text { steigender Ressourcenpreise }\end{array}$ \\
\hline Sicherheit & $\begin{array}{l}\text { - Zunehmender Datenaustausch erhöht } \\
\text { Risiken: } \\
\text { - Schutz von sensiblen Daten } \\
\text { - Cyber-Angriffe erhöhen sich } \\
\text { - Viele Netzwerke sind unzureichend } \\
\text { geschützt }\end{array}$ & $\begin{array}{l}\text { Dezentrale Vernetzung von } \\
\text { mehreren dutzenden/hunderten } \\
\text { Geräten maximiert die Sicherheit } \\
\text { - Lokale Verschlüsselung }\end{array}$ \\
\hline
\end{tabular}

Quelle: eigene Darstellung

Bei ressourcenintensiven Datentransporten wird hingegen das Konzept des Cloudlet Computing favorisiert (Fernández-Caramés et al. 2018). Weiter kann AR beim „Remote Live Support“ Anwendung finden. Oftmals wird für die mobilen Geräte das Konzept des MEC verwendet (Sprecher 2016). Edge Computing ermöglicht die gefilterte Erfassung von störungskritischen Daten, welche in Echtzeit analysiert werden können. Im Anschluss kann die Anleitung zur Störungsbehebung via Fernwartung dem bzw. der in der Fabrikhalle anwesenden Mitarbeitenden über das HMD eingeblendet werden (Scheer 2013; Schneider et al. 2017). Auch für die Bereiche Automated Inspection, Streaming Analytics oder das Condition Monitoring kann EC angewendet werden, da die Qualitätskontrolle des Menschen oftmals zu wenig schnell und fehlerbehaftet abgewickelt wird. Augmented Reality kann hier die Kontrollen noch präziser, zuverlässiger realisieren und in einem Bruchteil der Zeit verarbeiten (MICROSCAN 2017; Drichel 2008; Mennigen 2017; Satyanarayanan 2017). Abschließend können die gesammelten Daten der Fertigungsmaschinen auf einem Edge Device mithilfe von statistischen Analysefunktionen und Algorithmen ausgewertet werden. Daraus entstehen Diagnose- und Prognosemodelle, um Wartungen einerseits zeitgerecht vorherzusagen und die Auswirkungen von Ausfällen detailliert darzulegen (Wu et al. 2017). 


\subsection{Ziele und Methodik der Studie}

Ein zentrales Ziel der vorliegenden Studie war die Herausarbeitung eines theoretischen sowie empirisch-explorativ ermittelten Standes des Konzeptes sowie der Anwendung von Edge Computing in der Schweizer Fertigungsindustrie. Im Kern sollten das technische Verständnis, Geschäftsmodelle und Anwendungsszenarien, praktische Umsetzungen im Sinne der Pilotierung und des Roll-outs vom Proof of Concept, als auch Herausforderungen sowie Risiken analysiert und herausgearbeitet werden.

Die Theorie wurde durch ein deduktives Vorgehen, anhand von qualitativen Interviews durch Experteninterviews abgefragt und ein Abgleich zwischen Theorie und Praxis realisiert (Mayring 2002, S. 114 ff.). Dies, um zu eruieren, ob und wie Edge Computing in der Schweizer Fertigungsindustrie Anwendung findet und wie ,realitätsnah“ die aufgearbeitete Theorie ist.

Ziel dieser Experteninterviews war es, die Sichtweisen von 3 verschiedenen Bezugsgruppen, zu vereinen, um so gestützt eine Gegenüberstellung vorzunehmen. Bei den Gruppen handelt es sich um: (1) ICT-Experten (Informatiksicht: I), (2) Unternehmensberater (Consultant-Sicht: C) und (3) Fertigungsunternehmen (Unternehmenssicht: U). Für die Empirie konnten Experten folgender Unternehmen akquiriert werden:

- I: Swisscom (Schweiz) AG

- C: iSolutions

- C: itelligence AG

- U: Die Schweizerische Post

- U: Güdel Group AG

- U: RUAG Holding AG

- U: Ypsomed AG

Die Interviews dauerten jeweils 1,5 Stunden und erfolgten immer beim Experten respektive im Unternehmen, um teilweise auch Anlagen, Maschinen und Technologien zu besichtigen. Im Anschluss der Durchführung wurden die aufgezeichneten Interviews Wort für Wort transkribiert und nach dem Verfahren der Inhaltsanalyse codiert (Hussy et al. 2013, S. 255 ff.; Diekmann 2017, S. 576, 579 ff.; Mayring 2002, S. 114 ff.).

Ein systematisches und validiertes Vorgehen mit einem induktiven, präzisen Kategoriensystem ermöglichte es, die jeweils unterschiedlichen Interviews zu verarbeiten und gezielt Erkenntnisse daraus zu gewinnen. Die so entstandenen Kondensate wurden nach einer Qualitätsprüfung (Mehraugenprinzip und Diskussion im Plenum) weiterverarbeitet und geclustert. So entstanden final 3 Clusterdokumente: die Informatik- (I), die Consultant- (C) und die Unternehmenssicht (U). Die folgende Abb. 13.3 gibt einen Überblick über den gesamten Prozess. 


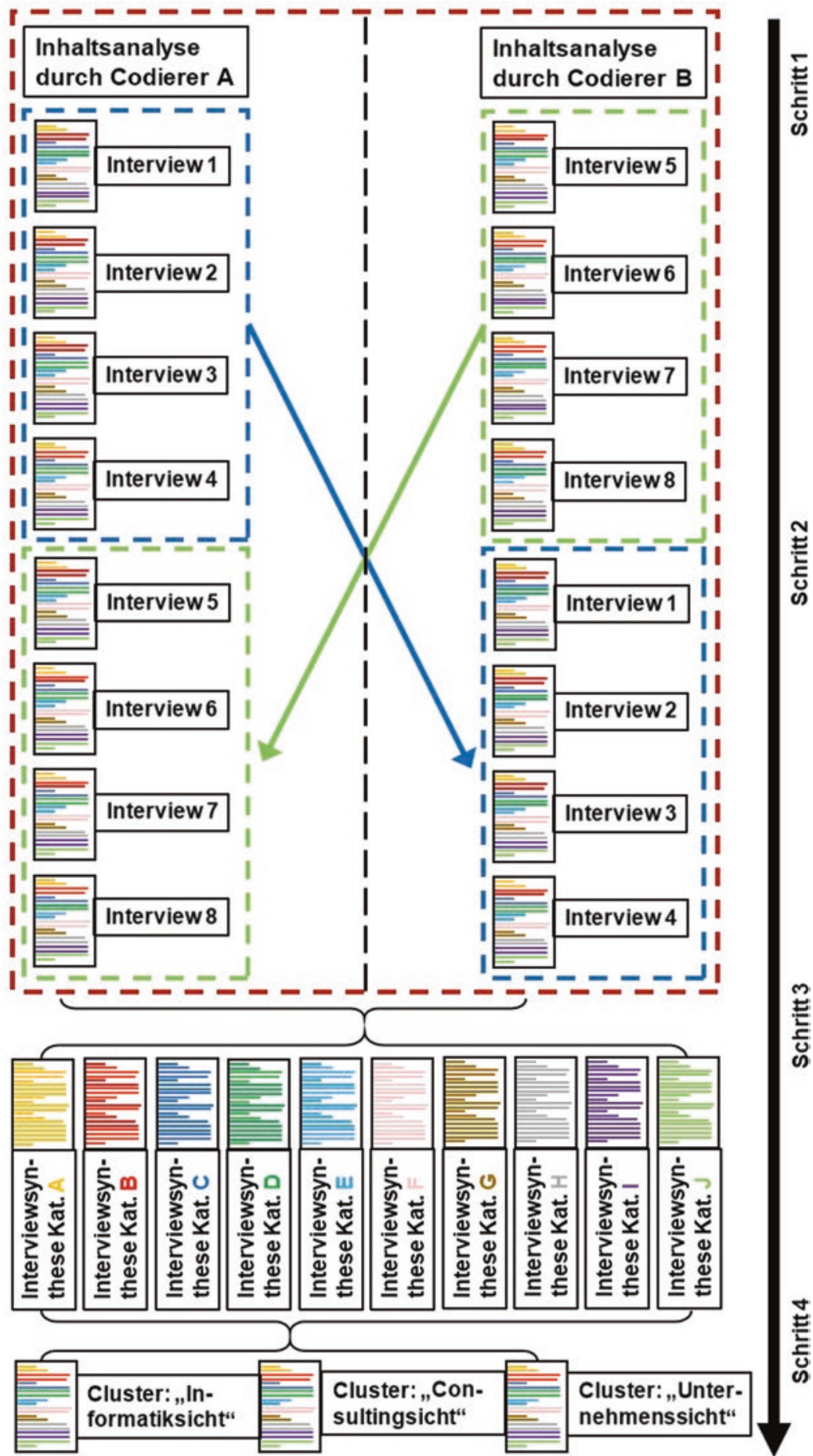

Abb. 13.3 Prozessablauf der definierten Inhaltsanalyse. (Quelle: eigene Darstellung) 


\subsection{Ergebnisse der Studie}

Nachfolgend dargestellt sind die zusammengefassten Erkenntnisse der Studie auf Basis der in der Zielsetzung definierten Themenkategorien.

\subsubsection{Technisches Verständnis}

Der Designansatz Edge Computing wird in der Praxis als kein neues Konzept verstanden, vielmehr integriert er sich in bestehende IT-Unternehmensarchitekturen. Fachexperten waren sich indessen einig, dass das Modell mit einer dezentralen Datenaggregation und einer client-basierten Datenverarbeitung direkt in der Nähe der Datenentstehung eingesetzt wird und somit Daten in „Information“" und „Wissen“ transformiert. Die Fertigungsindustrie stellt hierfür IT-Ressourcen an der lokalen Netzwerkkante, möglichst nahe bei der Maschine oder Anlage, zur Verfügung. Die Zielsetzung der Unternehmen sollte dabei beinhalten, dass zum Schluss die IT-Ressourcen eine rechnergestützte, selbstständige Intelligenz und die Einspielung von Algorithmen auf dem Edge Device ermöglichen sollen. Daraus können bereits in der Werkhalle technologische Standards von Business Intelligence erreicht werden. Dies, um beispielsweise datenbasiert Entscheidungen zu verbessern. Zum heutigen Betrachtungszeitpunkt wird jedoch das Edge Device vorwiegend als Datenfilterungskomponente und noch nicht als selbstständiger Logikträger oder als autonome Steuerungskomponente eingesetzt.

Damit EC funktioniert, braucht es eine Bedarfsanalyse technischer Anforderungen an das Edge Device. Die Bereitstellung von IT-Ressourcen bedingt daher CPU (Rechenleistung) wie auch RAM (Arbeitsspeicher), aber auch weitere Speichersysteme und HardwareKomponenten wie Hard Drives und Server Blades. Ein hoher IT Load erfordert von den Fertigungsunternehmen, viel CPU auf den Einheiten zur Verfügung zu stellen. Bei einem hohen Telco-Volumen steht die Übertragungsgeschwindigkeit (Gbit) im Fokus. Die einleitend erwähnten technischen Anforderungen werden über ein Edge Device gedeckt. Stand heute kann das Edge Device in der Ausprägung eines Edge Servers zur Anwendung kommen, jedoch gibt es auch Embedded Systems innerhalb der Fertigungsmaschine, Gateways, aber vereinzelt auch sehr performante Sensoren. Handelsübliche Sensoren können jedoch, mangels Leistungsfähigkeit, nicht als Edge Device definiert werden. Um der Leistungsanforderung gerecht zu werden, kommen öfters auch performantere, industrietaugliche Mikrocontroller von Anbietern wie Siemens oder ABB zum Einsatz.

Da die Sensoren und Aktoren weder autonome Steuerungskomponenten noch selbstständige Logikträger sind, wird die Steuerung des Anlagenparks auch nicht durch Interaktionen dieser sichergestellt. Dafür werden weiterhin industrielle Standards in Form der speicherprogrammierbaren Steuerung (SPS), selten auch Industriecomputer, verwendet. Die SPS ist eine verkabelte (LAN/analog), industriespezifische Zentrale, welche auf Basis der gesammelten Maschinen- und Sensordaten die jeweiligen Maßnahmen an die Ak- 
toren befiehlt. Eine weitere Individualität besteht in der Sensorik. Fertigungsanlagen sind oftmals Einzelanfertigungen, ausgestattet mit einer hohen Anzahl an Sensoren oder gar Feldbussystemen. Aufgrund ihrer Spezifika sind mehrheitlich ältere, industriegetriebene Kommunikationsprotokolle, wie beispielsweise Euro-Map 63, im Einsatz. Das Euromap-63-Protokoll ist auf eine Datenverarbeitung in der SPS ausgerichtet und entspricht oftmals nicht dem Kommunikationsstandard neuartiger Technologien. Damit diese Komptabilität zwischen von Edge Computing verwendeten Technologien und den Einzelfertigungsanlagen gegeben ist, müssen industrietaugliche Standardprotokolle wie OPC UA und IO.Link weiter vorangetrieben werden. Ebenso gilt dies für industriespezifische Transporttechnologien wie Bluetooth Low Energy und Wifi IO.11, aber auch KATM, LTM und NVIOT (4G Kompatibel), welche heute oft in der Industrie Anwendung finden. Die Wahl der richtigen Transporttechnologie entscheidet sich aus einem Trade-off zwischen Batterie bzw. Energieeffizienz, Kosten, Laufzeit, Reichweite und Geschwindigkeit. Die stetige Entwicklung dieser Schnittstellenstandards zwischen Smart Object und dem Daten-Aggregator (SPS, Edge Device) ist für die praxistaugliche Anwendung von EC von höchster Wichtigkeit, auch wenn bei den Transporttechnologien in der Praxis häufig die „Wired-Lösung“ mittels Ethernet oder Glasfaser-Anschluss bevorzugt wird.

Edge Computing kann in der industriellen Praxis jedoch erst dann Anwendung finden, wenn gesammelte Sensordaten direkt von der SPS abgezogen und auf einem naheliegenden Edge Server als Massendaten bereitgestellt werden. Auf diesem Edge Server können die Daten zur Analyse von Events und Maschinenzustandsinformationen verarbeitet werden. Ein weiterer Vorteil besteht darin, dass die Daten bereits auf dem Edge Server an der Netzwerkkante verschlüsselt und im Anschluss an weitere Umsysteme wie SAP/ERP, MES, CRM, aber auch an andere kognitive und analytische Applikationen weitergeleitet werden können. Dies ist in der Praxis noch nicht angekommen. Vermutet wird hier die Schwierigkeit, ausreichend Zeit, Budget und Know-how bereitzustellen.

Im Rahmen der (explorativen) Studie konnten keine Praxisanwendungen für die Architekturmodelle von Fog Computing und Cloudlet Computing gewonnen werden. Im Gegenzug hat das Konzept von Mobile Edge Computing in der Fertigungsindustrie bereits Einzug gefunden und wird in ersten Pilotprojekten realisiert. Das Architekturmodell kann in unterschiedlichen Ausprägungen realisiert werden. In Abb. 13.4 werden 4 Ansätze vorgestellt, wie die MEC-Server und Basisstationen positioniert werden.

Mobile Edge Computing kann somit je nach Bedarf und Situation über Distanz (1), an der Arealgrenze (2), im Campus (3) oder direkt in der Werkhalle (4) an den Maschinen platziert werden. Dabei bedient sich MEC diverser Übertragungsmöglichkeiten (4G/LTE, 5G) und ermöglicht mobilen Devices, wie auch nichtmobilen Devices, eine Verbindung zum Netzwerk. Hierbei können Daten vor dem Weitertransport bereits vorverschlüsselt werden (4). Der Einsatz von MEC stellt Echtzeitanforderungen sowie einen schnellen Datentransport zwischen den Endgeräten und dem MEC-Server sicher. Die Einführung von $5 \mathrm{G}$ verspricht hier eine weitere Verbesserung der Latenz. 


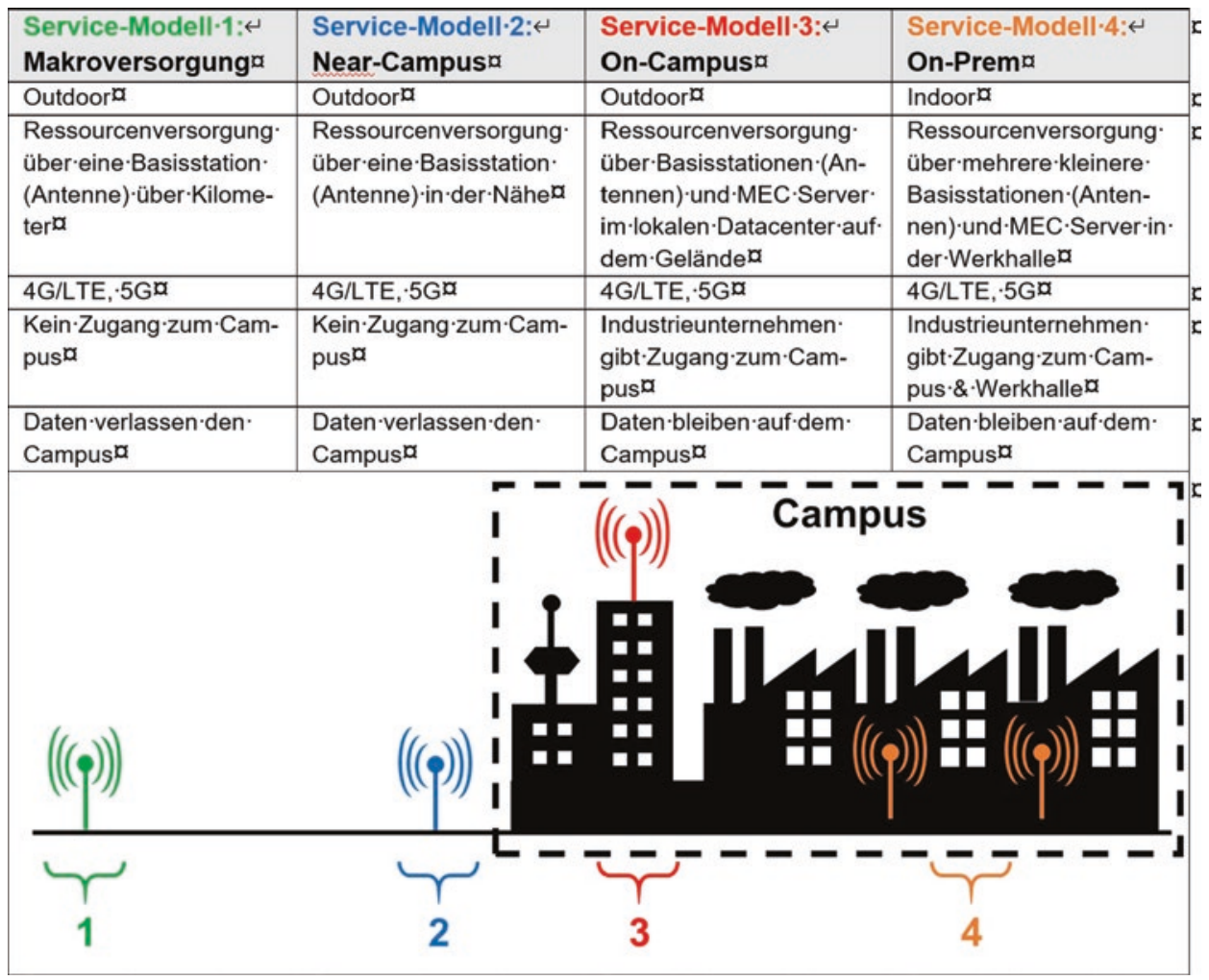

Abb. 13.4 Mobile-Edge-Computing-Anwendungsszenarien. (Quelle: eigene Darstellung)

\subsubsection{Geschäftsmodelle und Anwendungsszenarien}

Im Rahmen der Studie hielten die Fachexperten den aktuellen Entwicklungsstand von Edge Computing im Hype-Cycle-Modell von Gartner fest und äußerten sich zudem über die notwendige Zeitdauer bis zum Erreichen des Produktivitätsplateaus. Die Ergebnisse wurden in Abb. 13.5 zusammengefasst.

Die Fachgruppe war sich einig, dass sich Edge Computing noch im Stadium „Innovation Trigger“ oder „Peak of inflated expectations“ befindet. Das Architekturkonzept ist zum heutigen Betrachtungszeitpunkt noch nicht ausgereift, was zu seltenen Anwendungsfällen und nur mäßig entwickelten Geschäftsmodellen führt. Vielmehr überwiegt in der Praxis die Forschung für den Einsatz von EC, operativ umgesetzt werden die erzielten Forschungsergebnisse in der Folge dann erst in kleineren „Proof of Concepts“. Ein weiterer entscheidender Faktor für die seltenen praktischen Anwendungsfälle von EC findet sich in der unmittelbaren Abhängigkeit zum IoT. Auch das Konzept von IoT hat den Reifegrad für den Markt noch nicht erreicht und ordnet sich ebenfalls in der Phase „Innovation Trigger“ ein. Weitere Abhängigkeiten sind auch zur künstlichen Intelligenz 


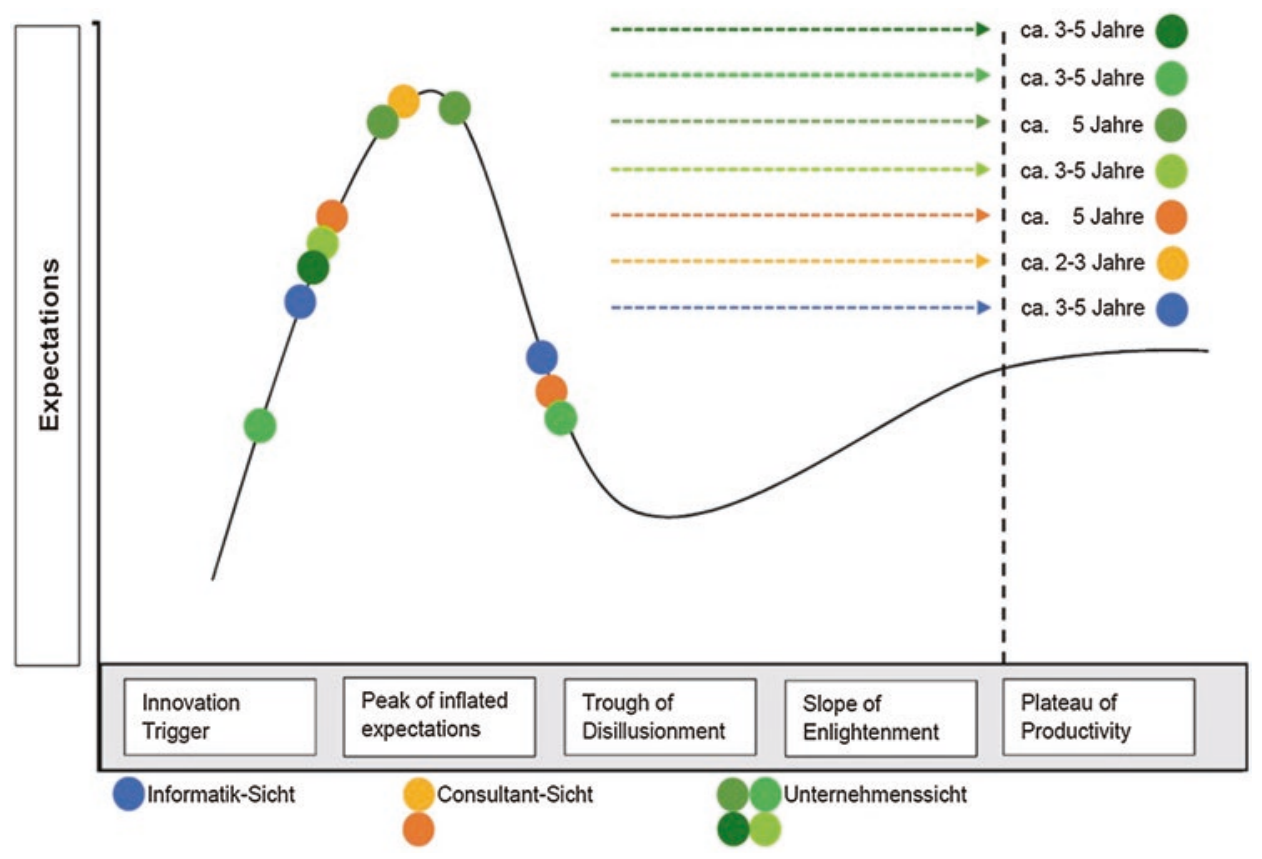

Abb. 13.5 Experteneinschätzung über den Stand heute und das Erreichen des „Plateau of Productivity“ von Edge Computing. (Quelle: eigene Darstellung in Anlehnung an Panetta 2017)

(KI) festzustellen. Künstliche Intelligenz kann zwar in Kombination mit Robotic Process Automation bereits repetitive Prozessvorgänge und einfache Entscheidungen übernehmen, die hohe Komplexität der Industrieprozesse erschwert jedoch die breitflächige Anwendung von $\mathrm{KI}$ in Kombination mit EC in der Fertigungsindustrie. Eine autonome Kommunikation zwischen Maschinen mithilfe von EC und IoT ist nach heutiger Einschätzung ein Zukunftsszenario, kann aber in der heutigen Ausgangslage und unter Berücksichtigung der technologischen Entwicklung noch nicht mit vernünftigem Aufwand umgesetzt werden.

Weiter haben die Forschungsergebnisse gezeigt, dass ICT-Anbieter mit der Zielgruppe „Industrie“ nicht das Konzept EC als Designansatz ,verkaufen“. Vielmehr steht die Effizienzsteigerung durch Digitalisierung im Vordergrund der Marketingkampagne. In der Verkaufsstrategie ist dabei die technologische Komponente oft zweitrangig und technologie-agnostisch. ICT-Anbieter verkaufen diese Leistungen mehrheitlich unter der IT-Outsourcing-Strategie, wobei die Unternehmen einzelne Komponenten oder die IT vollständig an einen IT-Provider auslagern. Setzt der IT-Provider im Rahmen eines IT-Outsourcings das Konzept von EC ein, stellt er Connectivity- oder IT-Ressourcen den Fertigungsunternehmen zur Verfügung. Als weiteres Geschäftsmodell kann ein ICT-Anbieter das Deployment in Betracht ziehen. Dabei können ,eintrainierte“ Algorithmen, beispielsweise aus der Azure Edge Cloud, direkt auf den Edge Devices eingespielt werden. Die 
Provider arbeiten intensiv an weiteren Forschungen im Rahmen des Deployments für EC und vermehrt werden Marketingaktivitäten von größeren IT-Providern darauf ausgerichtet.

Die Fertigungsindustrie kennt durch die Forschung und den bereits getätigten Piloten unterschiedliche Geschäftsmodelle, welche mithilfe von Edge Computing realisiert werden können. Ein naheliegendes Anwendungsszenario ist das Condition Monitoring (Fertigungsqualität, Sicherheit und Betriebskostenminimierung). Mithilfe der gemessenen Maschinendaten können Produktionsprozesse in Echtzeit abgebildet werden. Damit lassen sich Zustände, „Gesundheit“ und die Nutzungsintensität der Fertigungsmaschinen abbilden. Dabei gilt es zu beachten, dass durch die Anwendung von Condition Monitoring erhebliche Datenpools entstehen, welche am besten durch die Einspielung lokaler Algorithmen zur lokalen Filterung von irrelevanten Rohdaten minimiert werden. Durch den Einsatz von kognitiven Instrumenten und Image Recognition können die Unternehmen die Dimension der Datennutzung erweitern und zeitgleich einerseits Rückschlüsse zu fehlerhaften Fertigungen ziehen, anderseits auch den Ausschuss durch gezielte Maßnahmen minimieren.

Besonderes Potenzial erbringt Edge Computing im Zusammenhang mit proaktiven Wartungssystemen. Einem Stillstand des Anlageparks folgen oft hohe Ausschusskosten, welche es für jedes Fertigungsunternehmen zu vermeiden gilt. Besitzt ein Fertigungsunternehmen die Fähigkeit, mithilfe der Datenanalytik Muster zu erkennen, kann in einem 1. Schritt ein vorbeugendes Wartungssystem (Preventive Maintenance) realisiert werden. Dabei bildet Predictive Maintenance den weiteren, übergeordneten Schritt zur Erweiterung von Preventive Maintenance (Condition Monitoring als Basis.) Diese (grundlegenden) Fähigkeiten haben Unternehmen bereits erlangt und Preventive Maintenance wird in der Fertigungsindustrie bereits angewendet (Fertigungsqualität und Betriebskostenminimierung). Würde es den Unternehmen gelingen, in Echtzeit die gesammelten Sensordaten auf dem Edge Device auszuwerten, könnte im Störungsfall rechtzeitig im Fertigungsprozess interveniert und automatische Wartungsfenster ohne Folgeschäden durch unbrauchbaren Ausschuss durchgeführt werden. Ein weiterer ökonomischer Vorteil liegt in den niedrigen Wartungskosten. Denn ein in Echtzeit abgestimmter Wartungsplan hätte demnach für die Unternehmen eine deutliche Kostenreduktion zur Folge. Die Digitalisierung eines Fertigungsunternehmens mithilfe von EC ermöglicht nebstdem auch personalspezifische Möglichkeiten, um das Unternehmen effizienter zu organisieren (Agilität \& Flexibilität, Fertigungsqualität und Betriebskostenminimierung). Einerseits können mithilfe von Augmented Reality und den dafür notwendigen HoloLens-Brillen repetitive Tätigkeiten wie die Sortierung und Qualitätskontrolle effizienter ausgestaltet werden. Anstelle von einer händischen Mehrfachkontrolle (Vier-Augen-Prinzip) kann ein/e einzige/r Mitarbeitende/r mit einer HoloLens eine vollständige Qualitätskontrolle vornehmen. Dies da die Kamera jeden Prozessschritt mitüberwacht und aufzeichnet. Zudem können bürokratische Aufwände umgangen sowie Lifecycle-Daten eines Erzeugnisses direkt in den relevanten Umsystemen wie SAP/ERP und MES erfasst werden. Anderseits besteht die Möglichkeit, Wartung und Störungsbehebungen von Fertigungsanlagen über Fernzugriffe von Maschineningenieuren zu ermöglichen. Dies um den Status, die Maschinenaktivitäten und 
die Vergangenheitsprotokolle einzusehen. Zeitintensive Vor-Ort-Interventionen werden so umgangen und zeitkritische Fehler aus der Ferne gelöst. Die Fernwartung kann zudem verstärkt mit Augmented Reality erfolgen. So werden dem Support Daten mittels HoloLens in Echtzeit zur Verfügung gestellt. Auch das Freelancing von freiberuflichen Servicetechnikern ist ein denkbares Supportszenario, um lokal ansässige Spezialisten anstelle von weitentfernten Fieldservice-Mitarbeitenden einzusetzen. Besonders Unternehmen mit internationalem Charakter können so die Kosten für OnSite-Intervention deutlicher minimieren.

In einem dynamischen Marktumfeld mit variablen Nachfragemengen und stets ändernden Bedürfnissen erscheint das Modell „Losgröße 1 - Sonderanfertigung“ als äußerst attraktiv (Agilität \& Flexibilität und Modellierung). Edge Computing ermöglicht durch die Erfassung der Fertigungsprozesse eine exaktere Mengenproduktion. Dies lässt eine individuellere, nachfrageorientiertere Fertigung zu, womit die Marktreaktionsfähigkeit deutlich verbessert wird. Als weiterer Vorteil ist hervorzuheben, dass die Lagerkosten durch individuelle Fertigung anstelle von Serienfertigung gesenkt werden können (Betriebskostenminimierung).

Durch die digitale Abbildung des Fertigungsprozesses und der erfassten Produkterzeugnisse entstehen aber auch kommerzielle Chancen im Produktdesign. Durch die Integration von Embedded Edge Systems können gefertigte Einheiten mit datengetriebenen Zusatzleistungen erweitert werden (Agilität \& Flexibilität, Fertigungsqualität und Modellierung). Dies führt durch die Erschaffung einer einzigartigen Kundenexperience auf dem Markt bestenfalls zu einem Marktalleinstellungsmerkmal (USP). Hervorzuheben sind in diesem Zusammenhang etablierte „Pay per usage“-Modelle, wobei die Relevanz von Edge Computing zur Realisierung einer solchen Kundenexperience nicht eindeutig eruiert werden kann.

Ein weiteres Szenario von Mietmodellen im Kontext der Industrie 4.0 und Edge Computing ist die Vermietung von Fertigungsanlagen auf Stundenbasis. Eine Abrechnung erfolgt dann nach effektivem Gebrauch. Die Anwendung eines solchen „As a Service“-Modells ist dann möglich, wenn die Nutzungszeiten mithilfe von Sensoren zuverlässig gemessen und rasch lokal ausgewertet werden können (Betriebskostenminimierung und Agilität \& Flexibilität).

In Abb. 13.6 werden der potenzielle Nutzen und die daraus resultierenden Chancen zusammenfassend aufgezeigt.

\subsubsection{Pilotierung und Roll-out im Proof of Concept}

Ein Proof of Concept ist eine agile Methode, mit der kleine Schritte realisiert und bestätigt werden. Dieses Vorgehen hat sich besonders auch für die Anwendung von Edge Computing bewährt. Dies, da das Konzept zum heutigen Betrachtungszeitpunkt mehrheitlich erst erforscht wird. Viele Unternehmen sammeln mithilfe von IoT Daten und analysieren die zugrundeliegenden Datenströme. Konkrete Anwendungsszenarien und Geschäftsmodelle 

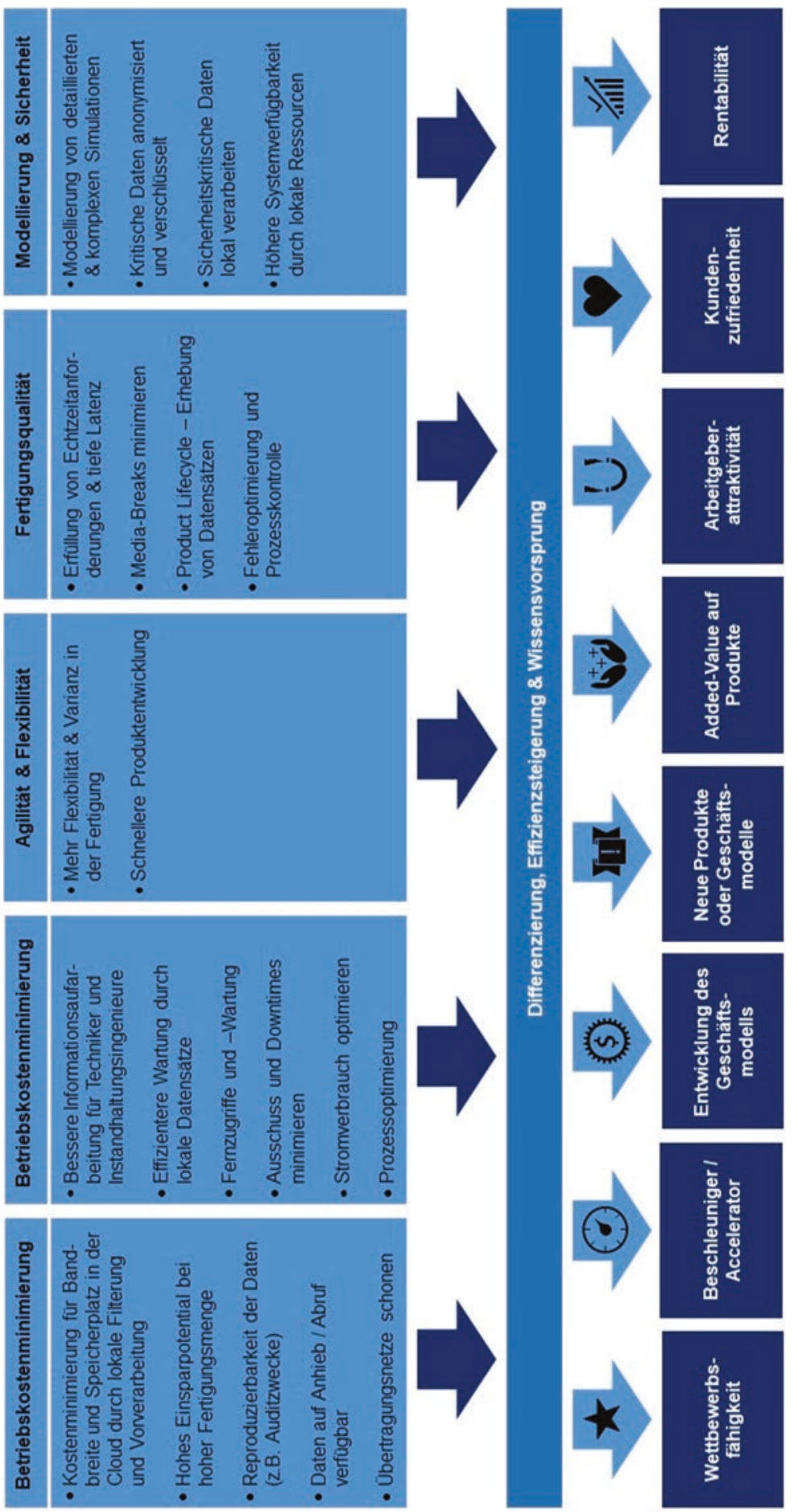

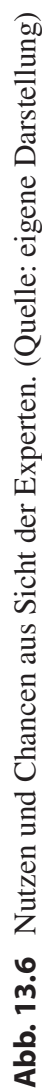
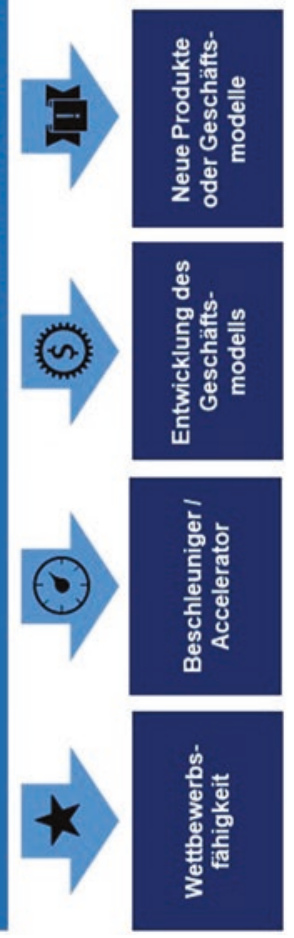
liegen selten vor, sodass auch nur wenige Unternehmen ihre Ziele durch die Anwendung von EC in einer digitalen Strategie festgehalten haben. Der Consulting Approach, wie in Abb. 13.7 dargestellt, legt den fertigenden Unternehmen nahe, dass bei der Anwendung von neuen Technologien folgende Vorgehensweise schrittweise durchlaufen wird.

In Abb. 13.8 wird ein Pilotprojekt eines Schweizer Fertigungsunternehmens vorgestellt, welches mit einzelnen Proof of Concepts das Konzept von Mobile Edge Computing erfolgreich in die Praxis umgesetzt hat.

Für den vorliegenden Show Case existiert ein Anlagenpark mit unterschiedlichen Spritzgussmaschinen. Diese Spritzgussmaschinen sind mit rund 2000 Sensoren vernetzt. Die ausgelesenen Sensordaten einer Maschine werden über LAN an die speicherprogrammierbare Steuerung (SPS) übergeben. Diese zentrale Einheit sammelt die Daten aller Sensoren und steuert zudem die Aktoren in Form von Befehlen an. Aus den 8 wichtigsten Datenaggregaten der Sensoren wird ein Datenabzug durchgeführt und über ein drahtloses Radio Access Network (RAN) auf dem MEC-Server bereitgestellt. Auf dem MEC-Server werden die Metadaten im Anschluss aufbereitet, normiert und zwischengespeichert. Im Folgeschritt werden die normierten Daten in einer Hadoop Cloud gespeichert und sortiert. Zum Schluss werden die Daten ausgewertet, für die Übermittlung aufbereitet und in Umsysteme, wie das Manufacturing Execution System (MES) und SAP/ERP, weitergeleitet.

\section{Consulting-Approach}

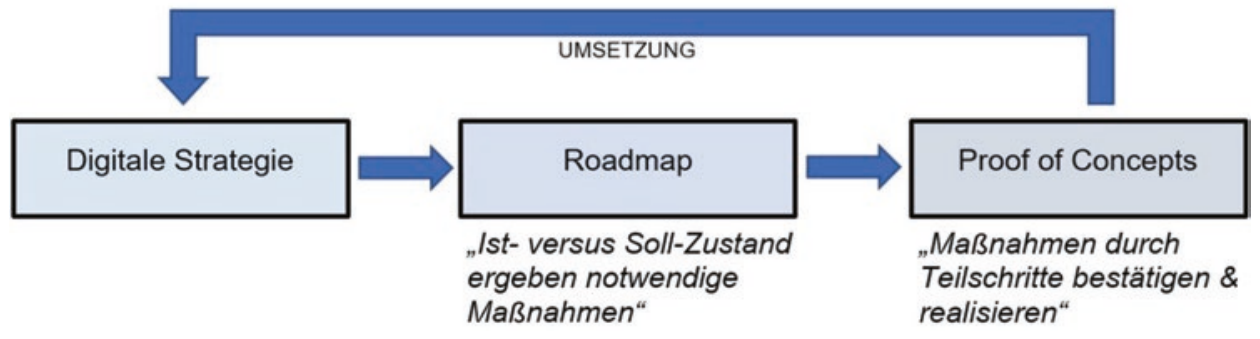

Abb. 13.7 Consulting Approach. (Quelle: eigene Darstellung)

\section{Show-Case Mobile Edge Computing}

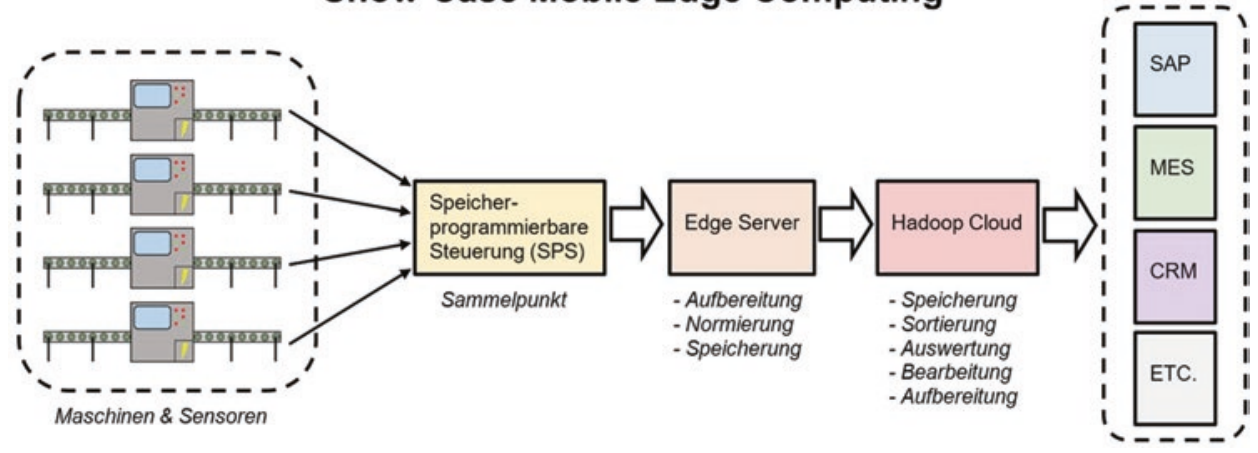

Abb. 13.8 Datenfluss Show Case Mobile Edge Computing. (Quelle: eigene Darstellung) 
Die Hadoop Cloud ist in diesem Show Case die zentrale Komponente, um Wartungsinterventionen (Preventive Maintenance) vorherzusagen und die Produktionsläufe auf Qualität zu prüfen. Ebenfalls bildet sie das Bindeglied zu den Umsystemen. So können die gewonnenen Datenaggregate die Logistikprozesse in SAP vervollständigt, anderseits wird die Fertigungsplanung im MES mit den aktuellen Fertigungsmengen und deren Qualität nachgeführt werden.

\subsubsection{Herausforderungen}

Tab. 13.3 soll eine Übersicht über die wichtigsten, praktischen Herausforderungen hinsichtlich der Anwendung von EC in der Fertigungsindustrie aufzeigen. Die Herausforderungen werden themenbezogen und nach ihrer Relevanz absteigend gegliedert.

Tab. 13.3 Herausforderungen durch Edge Computing in der Fertigungsindustrie

\begin{tabular}{|c|c|c|}
\hline Herausforderung & Ausprägungen der Herausforderungen & Relevanz \\
\hline $\begin{array}{l}\text { Wissen \& } \\
\text { Mindset }\end{array}$ & $\begin{array}{l}\text { - Rekrutierung von Fachspezialisten mit spezifischen } \\
\text { Kompetenzen in Zeiten eines Fachkräftemangels } \rightarrow \text { fordert eine } \\
\text { starke Rekrutierungspolitik } \\
\text { - Verständnis und Kompetenz für Digitalisierungsthemen und dem } \\
\text { damit verbundenen Potenzial fehlt oft } \rightarrow \text { Out of the Box } \\
\text { Thinking/,Entrepreneur-Gen“ fördern } \\
\text { - Agile Arbeitsweisen sind eine Grundvoraussetzung für Proof of } \\
\text { Concepts und damit verbundene hybride Architekturen } \rightarrow \\
\text { Erwerb der Fähigkeiten ist ein Muss } \\
\text { - Verschiebung von Tätigkeiten erfordert ein HR- } \\
\text { Changemanagement } \rightarrow \text { Entwicklung und Transformation durch } \\
\text { gezielte Förderung aktivieren } \\
\text { - Neuartige Designansätze und IT-Projekte sind oftmals von hoher } \\
\text { Komplexität } \rightarrow \text { Wissen muss aufgebaut und/oder durch Dritte } \\
\text { akquiriert werden } \\
\text { - Anbieter und Consulting-Unternehmen von EC-Konzepten } \\
\text { müssen Prozessverantwortung übernehmen } \rightarrow \text { Formulierung der } \\
\text { Verantwortlichkeiten ist ein Muss } \\
\text { - Digitalisierung bedingt Offenheit der gesamten Geschäftsleitung } \\
\rightarrow \text { Das gemeinsame Verständnis der Vision, Strategie und für das } \\
\text { gesetzte Ziel muss bekannt sein } \\
\text { - Transformationen führen zu grundlegenden Veränderungen } \rightarrow \\
\text { eine enge Führung unter Berücksichtigung der weichen Faktoren } \\
\text { ist unabdingbar } \\
\text { - Der Business Case und der damit verbundene Aufwand (Projekt } \\
\text { \& Betrieb) wird oftmals unterschätzt } \rightarrow \text { Wichtig ist die absolute } \\
\text { Transparenz der Kosten } \\
\text { - Wissen in neuen Disziplinen wie Produktmanagement und } \\
\text { Business Process Modelling sind notwendig } \rightarrow \text { Wissensaufbau } \\
\text { muss frühzeitig erfolgen }\end{array}$ & ++++ \\
\hline
\end{tabular}


Tab. 13.3 (Fortsetzung)

\begin{tabular}{|c|c|c|}
\hline Herausforderung & Ausprägungen der Herausforderungen & Relevanz \\
\hline $\begin{array}{l}\text { Challenge „Big } \\
\text { Data“ }\end{array}$ & $\begin{array}{l}\text { - Vorhandene Netzkapazitäten stoßen durch die Vernetzung an } \\
\text { Grenzen } \rightarrow \text { Architekturanforderungen müssen vor der } \\
\text { Pilotierung definiert werden } \\
\text { - Datenerhebung findet im breiten Umfang statt, eine } \\
\text { Nutzentransformation durch gezielte Analyse der Daten fehlt } \rightarrow \\
\text { Identifikation der unternehmenswichtigen Daten durch eine } \\
\text { gezielte Analyse muss weiter pilotiert werden } \\
\text { - Autonome Entscheidungsprozesse können in ,near live“ noch } \\
\text { nicht abgebildet werden } \rightarrow \text { geht einher mit der Lösung der } \\
\text { übrigen Herausforderungen } \\
\text { - Die richtigen Softwarekomponenten müssen eingesetzt werden, } \\
\text { um Daten möglichst gewinnbringend zu analysieren } \rightarrow \\
\text { Requirements Engineering ist zentral } \\
\text { - Das Beziehungswissen der erhobenen Daten ist von hoher } \\
\text { Komplexität } \rightarrow \text { Die Messbarkeit der Abhängigkeiten muss } \\
\text { weiterhin vorangetrieben werden } \\
\text { - Ausgelesene Daten sind durch Maschinen-Individualitäten } \\
\text { oftmals nicht standardisiert } \rightarrow \text { Standardisierung der Datensätze } \\
\text { ist elementar für die weitere Analyse }\end{array}$ & +++ \\
\hline Investitionen & $\begin{array}{l}\text { - Investitionsposten für IT-Projekte sind oftmals klein } \rightarrow \\
\text { Verabschiedung von größeren Investitionsposten durch die } \\
\text { Geschäftsleitung ist für die Digitalisierung wichtig } \\
\text { - Die einmaligen Kosten für IT-Projekte sind oftmals hoch und der } \\
\text { Gewinn nicht direkt messbar } \rightarrow \text { ROI muss für größere } \\
\text { Projektbudgets klar ausformuliert sein } \\
\text { - Die Budgetierung plant die finanziellen Kapazitäten anderweitig } \\
\text { ein } \rightarrow \text { Projekte wie EC sind oftmals langfristige Investitionen, } \\
\text { strategische Vorteile müssen auf lange Sicht erkennbar und } \\
\text { erreichbar sein }\end{array}$ & ++ \\
\hline Security & $\begin{array}{l}\text { - Mit der Digitalisierung entstehen regulatorische } \\
\text { Rahmenbedingungen hinsichtlich Access Management und } \\
\text { Datenschutz } \rightarrow \text { Schutzkonzept muss ausgearbeitet werden } \\
\text { - Durch die Digitalisierung muss die Datenhoheit und Haftung im } \\
\text { Fall eines Verlusts geregelt werden } \rightarrow \text { Klare vertragliche } \\
\text { Abgrenzungen zwischen Unternehmen und Provider notwendig } \\
\text { - Eine integrale Sicherheitsarchitektur zwecks Verfügbarkeit, } \\
\text { Integrität, Vertraulichkeit und Nachvollziehbarkeit ist } \\
\text { erforderlich } \rightarrow \text { Identifikation geschäftsvertraulicher Daten }\end{array}$ & ++ \\
\hline
\end{tabular}

(Fortsetzung) 
Tab. 13.3 (Fortsetzung)

\begin{tabular}{|c|c|c|}
\hline Herausforderung & Ausprägungen der Herausforderungen & Relevanz \\
\hline Infrastruktur & $\begin{array}{l}\text { - Maschinelle Metalle und elektromagnetische Störquellen } \\
\text { reduzieren oftmals die drahtlose Transportfähigkeit von Daten } \rightarrow \\
\text { Bei Investitionen in neue Anlagen muss die IT-Architektursicht } \\
\text { zunehmend berücksichtigt werden } \\
\text { - Anlagen sind oftmals noch mit älteren Hardware- und Software- } \\
\text { Komponenten mit unterschiedlichen Firmware-Versionen } \\
\text { ausgestattet } \rightarrow \text { HW- und Software-Standards sind bei dem } \\
\text { Einkauf neuer Anlagen zu berücksichtigen, um die } \\
\text { Datengewinnung zu vereinfachen }\end{array}$ & + \\
\hline $\begin{array}{l}\text { Weitere } \\
\text { Heraus- } \\
\text { forderungen }\end{array}$ & $\begin{array}{l}\text { - Mit der zunehmenden Digitalisierung des Anlageparks muss die } \\
\text { Orchestration von SW-Updates sichergestellt sein } \rightarrow \text { Ablösung } \\
\text { von individuellen Deployment-Szenarien mittels Skripten durch } \\
\text { Plug \& Play-Funktionen und Docker-Images, verbunden mit } \\
\text { einem Effizienzgewinn } \\
\text { - Die individuellen Protokolle der Anlagen erschweren eine } \\
\text { ganzheitliche Digitalisierung des Anlageparks } \rightarrow \text { Förderung von } \\
\text { Standardisierungsinitiativen ist unverzichtbar }\end{array}$ & + \\
\hline
\end{tabular}

Quelle: eigene Darstellung

\subsubsection{Risiken}

Wie grundsätzlich bei jeder technischen Implementierung besteht immer ein Risiko, dass die Anwendung nicht auf Anhieb funktioniert. Desto wichtiger ist es, dass die Implementierung von EC mithilfe von Proof of Concepts und mit der erforderlichen Agilität erfolgt, um das Risiko und die damit verbundenen Investitionen durch die Realisierung kleiner Teilschritte zu minimieren. Neue Designansätze wie EC müssen zudem auf den eigenen Betrieb projiziert werden können, damit die richtigen Schlussfolgerungen gezogen werden. Dieser Zusammenhang und die Komplexität werden aus der Erfahrung oftmals unterschätzt. Ebenso ist hervorzuheben, dass Fertigungsunternehmen die Vielschichtigkeit von IT-Konzepten wie EC an sich in vielen Fällen unterschätzen. Dabei erscheinen die Vernetzung und die damit verbundene Datenaggregation oftmals als unkompliziert, jedoch verbirgt sich hierbei das Risiko der Verzettelung bei der Datenanalyse, da keine geeignete Methodik zur Komplexitätsreduktion vorliegt. Ein Folgerisiko ist dabei oftmals die Überschreitung des bereitgestellten Projektbudgets, da indirekte, interne Aufwände oftmals nicht umfänglich genug betrachtet werden. Als weiterer Risikofaktor kann das Partnermanagement identifiziert werden. So ist die Wahl der richtigen Softwarekomponenten von zentraler Bedeutung, denn Software beinhaltet oftmals Risiken in Form von Bugs, Sicherheitslücken. Dies kann als Folge „Downtimes“ nach sich ziehen. Weiterhin können Sicherheitsanforderungen (beispielsweise durch Industriespionage) und Produktionskapazitäten gefährdet werden. 
Ebenso sind die juristischen Risiken hinsichtlich der Datenhoheit und eines möglichen Datenverlusts, als vertragliche Haftungsbestimmungen im Rahmen des Partnermanagements zu definieren. Denn durch die Vernetzung des Anlagenparks verbinden sich unterschiedliche Partner bzw. Unternehmen im Fertigungsprozess, wodurch bei Ausfällen und Datenverlusten zwingend vertragliche Haftungsbestimmungen zwischen den Parteien benötigt werden. Gleiches gilt ebenfalls für Umsysteme zu anderen Dritten, auch hier sind notwendige Sicherheitskonzepte zu konzipieren und Haftungsbestimmungen zu vereinbaren.

Zuletzt kann erwähnt werden, dass die Fertigungsunternehmen im Werben um ITFachkräfte auf dem Arbeitsmarkt in direkter Konkurrenz zu IT-Unternehmen stehen. Hierbei besteht ein erhöhtes Risiko, dass die Fertigungsunternehmen das Wissen nicht akquirieren können und von anderen Branchen ,ausgebremst“" werden.

\subsection{Schlussfolgerungen}

Im Folgenden wird der Entwicklungsstand und der Fortschritt von EC mithilfe des HypeCycle-Modells für aufkommende Technologien identifiziert. Hierfür wird die Positionierung von Gartner aus dem Jahr 2017, wie auch die Einschätzung aus der vorliegenden Studie aus dem Jahr 2019 herangezogen und in Abb. 13.9 gegenübergestellt.

Wie Gartner mit seiner Positionierung im Jahr 2017 unterlegte, erzeugte das Innovationskonzept von EC großes Interesse beim Fachpublikum. Auch die Disruption Map von Deloitte prognostizierte im Jahr 2015 einen erheblichen digitalen Umbruch der Fertigungsindustrie bis in das Jahr 2020, durchaus auch mit Konzepten wie EC. Mit der vorliegenden Studie aus dem Jahr 2019 konnte festgestellt werden, dass das Konzept praktisch nur selten zur Anwendung kommt und sich dadurch nur marginal entwickelt hat. Weiter befinden sich viele Unternehmen noch in der Forschungs- und Beobachtungsphase, zu hohe Erwartungshaltungen und „Kinderkrankheiten“ des Konzeptes sind womöglich oftmals nicht sichtbar, da noch keine praktischen Erfahrungen mit dem Konzept vorliegen. Einzelne Unternehmen haben in der Rolle als Early Adopter erste Proof of Concepts initialisiert, und werden sich den erläuterten Herausforderungen (v. a. hinsichtlich des Wissensbedarfs, verbunden mit der Datenanalyse und dem Business Process Modelling) und teilweise den zu hohen Erwartungshaltungen bewusst. Mit dieser Ernüchterung gelangen sie in das Tal der Enttäuschung. Jedoch sind sich die Experten einig, dass die Lancierung von standardisierten EC-Angeboten noch 2-5 Jahre dauern wird.

Eine weitere Erkenntnis liegt in den branchen- und unternehmenseigenen Spezifika, welche eine Anwendung von standardisierten Konzepten wie EC erschweren. Die Konzepte wie MEC, Cloudlet Computing und Fog Computing sind daher keine allgemeingültigen „Blueprints“. Zum einen muss der generische Designansatz auf die individuellen Betriebsabläufe projiziert werden können, zum anderen sind die eingesetzten Anlagen branchenweit mehrheitlich Einzelanfertigungen und werden über Jahre amortisiert. Der lange Lifecycle und die unterschiedlichen Altersklassen der Anlagen führen dazu, dass 


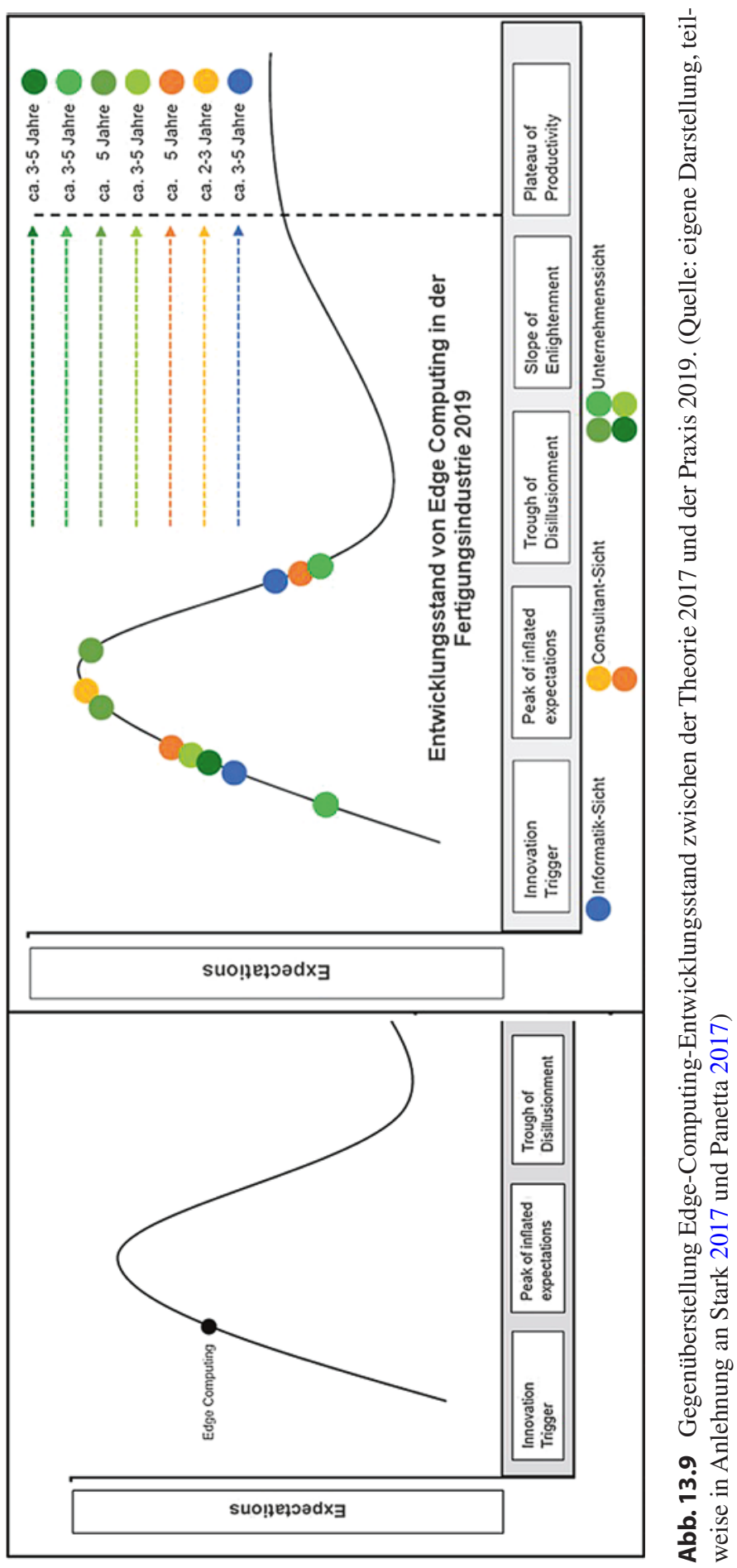


neuaufkommende, zwingend notwendige Standards noch nicht im Anlagepark verbaut sind. Dadurch ist die Datenverarbeitung weiterhin auf die SPS ausgerichtet und die verwendeten Kommunikationsprotokolle und Transporttechnologien entsprechen nicht den Grundanforderungen für eine digitale Transformation. Die Experten sind sich indessen einig, dass industriegetriebene Standardisierungsinitiativen eine zwingende Voraussetzung sind, damit der digitale Umbruch mithilfe von EC-Konzepten gelingen kann.

Hingegen konnte festgestellt werden, dass die Fachexperten aus dem Industriekonsortium bereits über ein grundlegendes Verständnis von EC verfügen. Besonders bei den möglichen Anwendungsszenarien von EC innerhalb der Fertigungsindustrie ist eine deutliche Übereinstimmung zu erkennen. So ist es nicht überraschend, dass die Early Adopter genau diese etablierten und bekannten Anwendungsszenarien in den Proof of Concepts bereits pilotiert haben. Gleicher Meinung war man auch hinsichtlich der Abhängigkeit zu anderen aufkommenden Technologien. Edge Computing ist im Grundsatz nur dann flächendeckend realisierbar, wenn IoT die vorausgesetzte Marktreife erlangt hat. Zudem wirken weitere aufkommende Technologien wie die Abbildung neuronaler Netze und das maschinelle Lernen mittels künstlicher Intelligenz beschleunigend auf die Entwicklung von EC ein. Im Gegensatz dazu gibt es unterschiedliche Aussagen zu den Funktionen innerhalb der Anwendung von EC. So gilt dies für manche EC als reine lokale Filter-, Normierungs- oder Analysekomponente für Massenrohdaten. Jedoch gab es auch entgegengesetzte Meinungen, dass EC immer mit einer Intelligenz und selbstlernenden Funktion verbunden ist.

Zuletzt gilt es die Anwendungsmöglichkeiten von EC hervorzuheben. Heutzutage wird in der Praxis öfters noch Preventive Maintenance betrieben. In naher Zukunft sollen auch erweiterte Wartungsmodelle wie Predictive Maintenance zur Anwendung kommen. Da EC zur Anwendung von Predictive Maintenance zwingend notwendig ist, wird die Entwicklung von EC in naher Zukunft weiterhin an Relevanz gewinnen. Weitere Szenarien wie die Pilotierung von Augmented Reality und der damit verbundene Effizienzgewinn im Bereich des Remote Supports, aber auch bei Qualitäts- und Sortiertätigkeiten, sind für die Wettbewerbsfähigkeit der Unternehmen zentral. Nicht überraschend werden genau die Bereiche in Unternehmen pilotiert, um die Digitalisierung und Automatisierung betriebsnotwendiger Prozesse mittels EC erheblich zu beschleunigen. Nebst dem marktgetriebenen Kostendruck nimmt auch die Verhandlungsmacht der Kunden stetig zu und erfordert individuelle Erzeugnisse. Die Kombination von Effizienzgewinnen bei gleichzeitiger Flexibilisierung der Produktionskette stellt für Unternehmen ein hohes Potenzial dar, so gilt es die Entwicklung dieser Pilotprojekte auf dem Markt mit hoher Aufmerksamkeit weiterzuverfolgen.

\subsection{Fazit und Ausblick}

Mit der vorliegenden Studie wurde eine komprimierte Darstellung über das Konzept Edge Computing geschaffen, welche einen ersten Einblick in dessen Funktionen und Anwendungsbereiche in der Schweizer Fertigungsindustrie liefert. Neben den 3 etablierten Archi- 
tekturansätzen liefern die aufgezeigten Ergebnisse der Studie einen Überblick hinsichtlich der Praxis und Anwendungsorientierung. Die unterschiedlichen Einschätzungen und Prognosen zum Entwicklungsstand von Edge Computing in der Fertigungsindustrie wie auch das Aufzeigen von potenziellen Chancen, Risiken, Herausforderungen, Diskrepanzen aber auch der damit verbundene Nutzen bieten Ansatzpunkte für weitere, tiefergehende Analysen.

In weiteren Forschungsarbeiten wäre wichtig zu ergründen, ob und wie die Architekturkonzepte in der Schweizer Fertigungsindustrie in den kommenden Jahren zur Anwendung kommen. Weiter bedarf es einer Beobachtung darüber, welche industriegetriebenen Standardisierungsbestrebungen für Protokolle, Maschinen und Technologien in Arbeit sind respektive welchen Mehrwert diese der Fertigungsindustrie und der Etablierung von Edge Computing der Branche geben können. Eine dedizierte Betrachtung der einzelnen Anwendungsszenarien wäre zudem sinnvoll, damit die Relevanz von Edge Computing detailliert für die Fertigungsindustrie der Schweiz aufgezeigt werden kann. Es wäre interessant in rund 5 Jahren, wenn Edge Computing das Produktivitätsplateau erreichen könnte, eine erneute Analyse der Situation vorzunehmen.

\section{Literatur}

1\&1 IONOS SE. (2018). Edge-Computing - Rechnen am Rand des Netzwerks. (12.06.2018, 1. I. SE, Hrsg.). https://www.ionos.de/digitalguide/server/knowhow/edge-computing-erklaerung-und-def inition/. Zugegriffen am 25.02.2019.

Abu Sharkh, M., Shami, A., \& Kalil, M. (2019). Edge computing - From hype to reality. In F. Al-Turjman (Hrsg.), Edge computing - From hype to reality. Antalya: Springer Nature. https://doi. org/10.1007/978-3-319-99061-3. Zugegriffen am 21.03.2019.

Borcoci, E., \& Obreja, S. (2018). Edge computing architectures - A survey on convergence of solutions (18.02.2018, T. T. Applications, Hrsg.). thinkmind.org: https://www.thinkmind.org/index. php?view=article\&articleid=future_computing_2018_1_50_30099. Zugegriffen am 24.03.2019.

Brüning, A., Dürr, C., Grossmann, C., Haderer, K., Hentscholek, W., Herold, T. (2017). Digital Engineering Magazin 1/17 (12.04.2017, H.-J. Grohmann, Hrsg., \& R. Trummer, Redakteur). Vaterstetten/München: WIN-Verlag GmbH \& Co. KG. https://www.digital-engineering-magazin.de/ sites/default/files/magazine-pdf/de_2017-01_archiv_reduziert.pdf. Zugegriffen am 04.04.2019.

DATACOM Buchverlag GmbH. (2017). Edge device (19.07.2017). itwissen.info: https://www.itwissen.info/edge-device-Edge-Device.html. Zugegriffen am 06.03.2019.

Diekmann, A. (2017). Empirische Sozialforschung - Grundlagen, Methoden, Anwendungen (11. A. Ausg.). (R. T. Verlag, Hrsg.). Hamburg: Rowohlt Taschenbuch. Zugegriffen am 13.04.2019.

Dolui, K., \& Kanti Datta, S. (2017). Comparison of edge computing implementations: Fog computing, cloudlet and mobile edge computing (IEEE, Hrsg.). https://doi.org/10.1109/GIOTS.2017.8016213. Zugegriffen am 04.03.2019.

Drichel, A. (2008). Realisierung eines flexiblen Klassifikators für die Industrielle Bildverarbeitung (15.05.2008, T. F. Bielefeld, Hrsg.). Bielefeld. http://www.4meshop.de/daten/Diplomarbeit.pdf. Zugegriffen am 09.09.2019.

El-Sayed, H., Sankar, S., Mukesh, P., Deepak, P., Akshansh, G., Manoranjan, M., \& Lin, C.-T. (2018). ieeexplore.ieee.org (IEEE 14.02.2018, Hrsg.). ieeexplore.ieee.org: https://ieeexplore.ieee.org/ stamp/stamp.jsp?arnumber=8166730. Zugegriffen am 05.03.2019. 
Eppenberger Digital. (2019). Willkommen in der digitalen Revolution. eppenbergerdigital.com: https://www.eppenbergerdigital.com/de/iot-internet-of-things/. Zugegriffen am 10.04.2019.

EUROFORUM Schweiz AG. (2019). BRANCHENTREFF ZUM JAHRESAUFTAKT - 5. Schweizer Strategietag Industrie 4.0. euroforum.ch: https://www.euroforum.ch/strategietagung/\#. Zugegriffen am 03.03.2019.

Fernández-Caramés, T. M., Fraga-Lamas, P., Suárez-Albela, M., \& Vilar-Montesinos, M. (2018). A fog computing and cloudlet based augmented reality system for the industry 4.0 Shipyard (02.06.2018, M. AG, Hrsg.).https://doi.org/10.3390/s18061798. Zugegriffen am 10.03.2019.

Flörkemeier, F., \& Mattern, F. (2010). Vom Internet der Computer zum Internet der Dinge (Springer, Hrsg.). Zürich: Springer. https://doi.org/10.1007/s00287-010-0417-7. Zugegriffen am 04.03.2019.

Friedli, T., Budde, L., Benninghaus, C., Elbe, C., \& Remling, D. (2018). National Study 2018 - Swiss Manufacturing Survey. National Study, Swiss Manufacturing Survey, Personalized Report for General Report (29.06.2018). St. Gallen: Universität St. Gallen, Institut für Technologiemanagement. https://www.raiffeisen.ch/content/dam/www/rch/pdf/publikationen/Studien/de/SwissManufacturing-Survey-de.pdf. Zugegriffen am 10.03.2019.

Fujitsu Technology Solutions AG. (2017). Die Fertigungsindustrie sieht sich als Vorreiter der Digitalen Transformation (18.04.2017). http://www.fujitsu.com/ch/de/about/resources/news/pressreleases/2017/2017-04-18-die-fertigungsindustrie-sieht-sich-als-vorreiter.html. Zugegriffen am 10.04.2019.

Fuqaha, Guizani, M., Mehdi, M., Aledhari, M., \& Ayyash, M. (2015). Internet of things: A survey on enabling technologies, protocols, and applications (IEEE 15.06.2015, Hrsg.). IEEE. https://doi. org/10.1109/COMST.2015.2444095. Zugegriffen am 06.03.2019.

Gaggi, O., Manzani, P., Palazzi, C., Burjani, A., \& Marquez-Barja, J. (2017). Smart objects and technologies for social good. Smart Objects and Technologies for Social Good, (1) Venedig: Springer International Publishing. https://doi.org/10.1007/978-3-319-61949-1. Zugegriffen am 06.03.2019.

Goertz, C., \& Salge, M. (2019). Edge Computing ermöglicht innovative Anwendungsfälle (09.03.2019, D. I. GmbH, Herausgeber). https://www.detecon.com/de/wissen/edge-computingermoeglicht-innovative-anwendungsfaelle. Zugegriffen am 07.04.2019.

Gombos, A. (2019). Cloud-Computing, Fog-Computing und Edge-Computing. Vor- und Nachteile der Dezentralisierung in der Auswertung von Maschinendaten in Industrieanwendungen (18.02.2019, Sentin, Herausgeber). https://sentin.ai/archives/1659. Zugegriffen am 30.03.2019.

Gross, B. (2018). Kanten, Wolken und das IoT (25.06.2018, T. V. GmbH, Hrsg.). it-production.com: https://www.it-production.com/industrie-4-0-iot/edge-computing-cloud/.Zugegriffen am 29.03.2019.

Grosser, C. (2018). Digitalisierung ist die neue Normalität (04.09.2018, S. AG, Hrsg. \& Swisscom (Schweiz) AG). https://magazin.swisscom.ch/digitale-transformation/die-normalitat-heisstdigitalisierung/. Zugegriffen am 03.03.2019.

Harting, A., Kolev, N., Redweik, J., Boyd, P., Hiebeler, M., \& Condrea, A. (2015). Überlebensstrategie „Digital Leadership“ (D. D. Consultancy, Hrsg.). https://www2.deloitte.com/content/dam/ Deloitte/de/Documents/technology/Survival\%20through\%20Digital\%20Leadership_safe.pdf. Zugegriffen am 01.03.2019.

Hewlett Packard Enterprise. (2019). Was ist Edge Computing? (H. P. LP, Herausgeber, \& Hewlett Packard Enterprise Development LP). hpe.com: https://www.hpe.com/ch/de/what-is/edgecomputing.html. Zugegriffen am 01.02.2019.

Hoffmann, D. (2015). Schweizer Fertigungsindustrie - Ein Überblick (10.09.2015, E. P. Ltd, Hrsg., \& Ebury Partners UK Ltd). ebury.ch: https://www.ebury.ch/blog/2015/09/10/schweizerfertigungsindustrie/. Zugegriffen am 12.03.2019.

Hoti, S. (2019). Schöne neue vernetzte Welt. Schöne neue vernetzte Welt (04.04.2019, F. Swiss, Hrsg.). https://fokus.swiss/2019/04/04/schoene-neue-vernetzte-welt/. Zugegriffen am 07.03.2019. 
Hüning, F. (2018). Embedded Systems für IoT. Aachen: Springer Vieweg. https://doi.org/10.1007 1978-3-662-57901-5. Zugegriffen am 05.03.2019.

Hussy, W., Schreier, M., \& Echterhoff, G. (2013). Forschungsmethoden in Psychologie und Sozialwissenschaften für Bachelor (2. A. Ausg.). (S.-V. B. Heidelberg, Hrsg.). Berlin/Heidelberg: Springer. https://doi.org/10.1007/978-3-642-34362-9. Zugegriffen am 10.03.2019.

IFS Deutschland GmbH \& Co. KG/IFS Schweiz AG. (2019). https://www.ifsworld.com/de/. Zugegriffen am 18.02.2019.

IT Verlag für Informationstechnik GmbH. (2017). Rechenzentrum-Trends 2018: Was für IT-Manager jetzt wichtig ist (18.12.2017, IT Verlag für Informationstechnik GmbH). it-daily.net: https:// www.it-daily.net/it-management/data-center-server-storage/17494-rechenzentrum-was-fuer-itmanager-jetzt-wichtig-ist. Zugegriffen am 17.03.2019.

itelligence AG. (2018). Elemente einer IoT Strategie | Teil 1: Die Bedeutung einer IoT Strategie (17.01.2018). itelligencegroup.com: https://itelligencegroup.com/ch/local-blog/32232/. Zugegriffen am 18.02.2019.

Jansen, J. (07. Februar 2017). 8,4 Milliarden vernetzte Geräte im Internet der Dinge. Frankfurter Allgemeine Zeitung GmbH. faz.net: https://www.faz.net/aktuell/wirtschaft/netzwirtschaft/ digitalisierung-8-4-milliarden-vernetzte-geraete-im-internet-der-dinge-14865654.html. Zugegriffen am 22.04.2019.

Jung, T. (2017). Vergleich aktueller LPWAN-Technologien im Internet der Dinge unter Einbindung von Energy-Harvesting (H. Offenburg, Hrsg.). Offenburg. https://opus.hs-offenburg.de/frontdoor/deliver/index/docId/2297/file/TomJung_Bachelorthesis_final.pdf. Zugegriffen am 09.04.2019.

Kahl, J. (2017). IoT in Berlin (C. Dr. Hammel, Hrsg.). https://www.technologiestiftung-berlin.de/ fileadmin/daten/media/publikationen/170504_IoT-Report_Web.pdf. Zugegriffen am 16.03.2019.

Kithion, B. (2016). Actuators in IOT (29.03.2016). learniot.wordpress.com: https://learniot.wordpress.com/2016/03/29/actuators-in-iot/. Zugegriffen am 28.03.2019.

Lee, G., \& Crespi, N. (2011). Internet of things for smart objects: ubiquitous networking between humans and objects. Internet of things for smart objects: ubiquitous networking between humans and objects (I. C. Technology, Hrsg.). Wuhan. https://hal.archives-ouvertes.fr/hal-01465049/document. Zugegriffen am 11.04.2019.

Liu, S., Tekinerdogan, B., Aoyama, M., \& Zhang, L.-J. (2018). Edge Computing - EDGE 2018. Seattle: Springer International Publishing. https://doi.org/10.1007/978-3-319-94340-4. Zugegriffen am 03.03.2019.

Martins, F., \& Kobylinska, A. (2019). IoT-Basics: Was bedeutet Edge Computing? (25.01.2019). Vogel Communications Group. https://www.industry-of-things.de/iot-basics-was-bedeutetedge-computing-a-678225/. Zugegriffen am 20.02.2019.

Mayring, P. (2002). Einführung in die qualitative Sozialforschung. Weinheim: Verlagsgruppe Beltz, Julius Beltz GmbH \& Co. KG. https://www.beltz.de/fachmedien/psychologie/buecher/produkt_ produktdetails/4329-einfuehrung_in_die_qualitative_sozialforschung.html. Zugegriffen am 13.04.2019.

Mell, P., \& Grance, T. (2011). The NIST definition of cloud computing - Recommendations of the National Institute of Standards and Technology (N. I. Technology, Hrsg.) http://faculty.winthrop. edu/domanm/csci411/Handouts/NIST.pdf. Zugegriffen am 13.04.2019.

Mennigen, M. (2017). Qualitätsmanagement in KMU - IT als wichtig(st)e Determinante (22.05.2017, web-malocher, Hrsg., \& itsystemkaufmann.de). https://www.itsystemkaufmann.de/qualitaetsmanagement-in-kmu-it-als-wichtigste-determinante/. Zugegriffen am 09.05.2019.

Microscan. (2017). Präzise Rückverfolgbarkeit und Inspektionslösungen für Automatisierungs- und OEM-Anwendungen. Microscan. https://www.bibus.ch/fileadmin/product_data/microscan/documents/DE_microscan_product_catalog.pdf. Zugegriffen am 09.05.2019. 
van Moorsel, W. (2016). Die Dinge im Internet. Die Dinge im Internet - NETZWERKARCHITEKTUR (AnyWebAG, Hrsg.) Zürich: AnyWeb AG. https://www.cnlab.ch/fileadmin/documents/Publikationen/2016/Die_Dinge_im_Internet_-_Netzwerkarchitektur.pdf. Zugegriffen am 26.01.2019.

Mouradin, C., Nabousli, D., Yangui, S., Glitho, R., Morrow, M., \& Polakos, P. (2017). A comprehensive survey on fog computing: Stateof-the-art and research challenges (IEEE 09.11.2017, Hrsg.). https://doi.org/10.1109/COMST.2017.2771153. Zugegriffen am 03.03.2019.

Mutschler, S. (2017). Edge Computing, Randerscheinung mit zentraler Bedeutung (18.09.2017). Golem Media GmbH. https://www.golem.de/news/edge-computing-randerscheinung-mitzentraler-bedeutung-1709-129248.html. Zugegriffen am 21.02.2019.

Najmul, H., Gillani, S., Ejaz, A., Ibrar, Y., \& Imran, M. (2018). The role of edge computing in the internet of things. The role of Edge Computing in the internet of things (29.08.2018, I. C. Magazine, Hrsg.). IEEE. https://doi.org/10.1109/MCOM.2018.1700906. Zugegriffen am 04.03.2019.

Neubäumer, R., Hewel, B., \& Lenk, T. (2017). Volkswirtschaftslehre. Grundlagen der Volkswirtschaftstheorie und Volkswirtschaftspolitik (6. Aufl.). Gabler. https://doi.org/10.1007/978-3-658-16523-9. Zugegriffen am 25.02.2019.

Obiltschnig, G. (2019a). Edge und Fog Computing Architekturen im Industrial IoT (10.03.2019). Edge und Fog Computing Architekturen im Industrial IoT. Maria Elend. https://www.appinf. com/download/EdgeFogIoT.pdf. Zugegriffen am 29.03.2019.

Obiltschnig, G. (2019b). Was steckt hinter dem Internet der Dinge? Was steckt hinter dem Internet der Dinge? Alles neu oder doch nur Embedded Business as usual? Maria Elend. https://www. appinf.com/download/InternetDerDinge.pdf. Zugegriffen am 27.03.2019.

Ostler, U. (2018). Der Computing-Dreiklang - aus jedem Eck und im Nebel - Ohne Glasfaser kein Edge Computing (27.09.2018). Vogel IT-Medien GmbH. https://www.datacenter-insider.de/ ohne-glasfaser-kein-edge-computing-a-759862/. Zugegriffen am 14.04.2019.

Panetta, K. (2017). Top trends in the gartner hype cycle for emerging technologies, 15.08.2017. Gartner, Inc. https://www.gartner.com/smarterwithgartner/top-trends-in-the-gartner-hype-cyclefor-emerging-technologies-2017/. Zugegriffen am 03.01.2019.

QSC AG. (2017). Edge-Computing warum das Prinzip Nähe schneller macht (04.01.2017). https:// digitales-wirtschaftswunder.de/edge-computing-warum-das-prinzip-naehe-schneller-macht/. Zugegriffen am 03.01.2019.

Reinsel, D., Gantz, J., \& Rydning, J. (2018). The digitization of the world - From edge to core (IDC, Hrsg.). Framingham. https://www.seagate.com/files/www-content/our-story/trends/files/idcseagate-dataage-whitepaper.pdf. Zugegriffen am 07.03.2019.

Rüdiger, A., \& Ostler, U. (2019). Was ist ein Cloudlet? (05.02.2019). Vogel IT-Medien GmbH. https:// www.datacenter-insider.de/was-ist-ein-cloudlet-a-795848/. Zugegriffen am 11.04.2019.

Rudolph, R. (2019). Industrie 4.0/Digitalisierung. (Swissmem). https://www.swissmem.ch/de/ industrie-politik/industrie-40-digitalisierung.html. Zugegriffen am 03.03.2019.

Sasson, D. (2019). An introduction to edge computing for IoT (01.02.2019). Hanu Software Solutions Inc. hanu.com: https://hanu.com/an-introduction-to-edge-computing-for-iot/. Zugegriffen am 12.02.2019.

Satyanarayanan, M. (2017). The emergence of edge computing (05.01.2017). IEEE. https://doi. org/10.1109/MC.2017.9. Zugegriffen am 03.03.2019.

Scheer, A.-W. (2013). Industrie 4.0 - Wie sehen Produktionsprozesse im Jahr 2020 aus? Industrie 4.0 - Wie sehen Produktionsprozesse im Jahr 2020 aus? (V. Zimmermann, I. Plaetrich, L. Wolf-Dietrich, \& T. Hausmann, Redakteure). Saarbrücken. https://www.researchgate.net/profile/August_Wilhelm_Scheer/publication/277717764_Industrie_40_-_Wie_sehen_Produktionsprozesse_im_Jahr_2020_aus/links/55ee9e5608ae0af8ee1 a1d72/Industrie-40-Wie-sehenProduktionsprozesse-im-Jahr-2020-aus.pdf. Zugegriffen am 09.05.2019. 
Schneider, M., Rambach, J., \& Stricker, D. (2017). Augmented reality based on edge computing using the example of remote live support. Augmented reality based on edge computing using the example of remote live support (IEEE 04.05.2017, Hrsg.). Toronto. https://doi.org/10.1109/ ICIT.2017.7915547. Zugegriffen am 14.03.2019.

Seeberg, P. (2016). Industrial Data Intelligence, Daten werden zum Produktionsfaktor (02.11.2016). Vogel IT-Medien GmbH. https://www.bigdata-insider.de/daten-werden-zum-produktionsfaktora-552121/. Zugegriffen am 22.02.2019.

Sprecher, N. (2016). Mobile edge computing a key technology towards $5 G$ (ETSI, Hrsg.). London. https://www.etsi.org/component/rsfiles/preview?path=MEC+Presentations+at+Industry+Event s\%5C201606+-+5G+World+-+ETSI+Mobile+Edge+Computing.pdf. Zugegriffen am 10.05.2019.

Stark, J. (2017). Diese drei Megatrends bestimmen laut Gartner die Digitalisierung (16.08.2017). Neue Mediengesellschaft Zürich AG. www.computerworld.ch: https://www.computerworld.ch/ business/hardware/drei-megatrends-bestimmen-laut-gartner-digitalisierung-1443324.html. Zugegriffen am 22.03.2019.

Steinhaus, I. (2018). 50 Milliarden vernetzte Geräte im Jahr 2022 (15.06.2018, IT-Zoom, Herausgeber, \& MEDIENHAUS Verlag GmbH). https://www.it-zoom.de/mobile-business/e/50milliarden-vernetzte-geraete-im-jahr-2022-19966/. Zugegriffen am 25.02.2019.

Stergiou, C., Psannis, K., Kim, B.-G., \& Gupta, B. (2016). Secure integration of internet-of-things and cloud computing (01.12.2016, Elsevier, Hrsg.). Elsevier B.V. https://doi.org/10.1016/j.future.2016.11.031. Zugegriffen am 07.03.2019.

Stiehler, A., \& Bieber, E. (2016). Moderne Arbeitsumgebungen in der Schweizer Industrie. Moderne Arbeitsumgebungen in der Schweizer Industrie-Anspruch und Wirklichkeit. (Swisscom(Schweiz) AG, Hrsg.). München. http://www.industrie2025.ch/fileadmin/user_upload/SwisscomModerne_ Arbeitsumgebungen-de.pdf. Zugegriffen am 10.03.2019.

Taleb, T., Sabella, D., Samdamis, K., Mada, B., Flinck, H., \& Dutta, S. (2017). On multi-access edge computing: A survey of the emerging $5 G$ network edge cloud architecture and orchestration (IEEE 18.05.2017, Hrsg.). IEEE. https://doi.org/10.1109/COMST.2017.2705720. Zugegriffen am 09.03.2019.

UTK Media GmbH. (2018). Diese Trends verändern 2018 die Fertigungsindustrie (11.01.2018). https://www.professional-computing.ch/diese-trends-veraendern-2018-die-fertigungsindustrie/. Zugegriffen am 18.02.2019.

Volk, M. (2014). Vor- und Nachteile der Nutzung von Cloud-Diensten (mit mobilen Endgeräten) in Organisationen und deren Einfluss auf die Nachhaltigkeit (02.06.2014). Magdeburg. http://bauhaus.cs.uni-magdeburg.de:8080/miscms.nsf/FEA8C8150500AA14C1257449004F79A 9/2903471F8768C568C1257DD4004DEDD1/\$FILE/Bachelorarbeit\%20Matthias\%20Volk.pdf. Zugegriffen am 01.03.2019.

Wu, D., Liu, S., Zhang, L., Terpenny, J., Gao, R., Kurfess, T., \& Guzzo, J. (2017). A fog computingbased framework for process monitoring and prognosis in cyber-manufacturing (01.04.2017, E. BV, Hrsg.). https://doi.org/10.1016/j.jmsy.2017.02.011. Zugegriffen am 20.03.2020.

Ziegler, P. A. (2017). Industrie 4.0 - noch fehlt es an Transparenz (18.10.2017, Netzwoche, Hrsg, \& Netzmedien AG). https://www.netzwoche.ch/studien/2017-10-18/industrie-40-noch-fehlt-es-antransparenz. Zugegriffen am 22.01.2019.

Zimmermann, W. (2016). Umbruch in der Chefetage - vom Heldentum zur agilen Führung. Umbruch in der Chefetage (1. Aufl.). Freiburg/München/Stuttgart: Haufe-Lexware GmbH \& Co. KG. https://books.google.ch/books?id=qDcKDQAAQBAJ\&pg=PA85\&lpg=PA85\&dq=Milliard en+vernetzte+objekte\&source=bl\&ots=-bt7sT_6fJ\&sig=ACfU3U3-ZOg5Pmj1 xdwCY97egRE R31BMsA\&hl=de\&sa=X\&ved=2ahUKEwjWuoirisPhAhUUw8QBHQYuDRc4ChDoATABegQIChAB\#v=onepage\&q=Milliarden\%20vernet. Zugegriffen am 10.04.2019. 
Appius, Dominik, Berufsbegleitendes Bachelorstudium in Betriebsökonomie an der Berner Fachhochschule mit Vertiefung in „Management in Practice: Strategy“. Seit mehreren Jahren bei der Swisscom (Schweiz) AG in verschiedenen Funktionen tätig. Aktuell in der Funktion als Management Consultant Banking und verantwortlich für die Pflege der Kundenbeziehung, kommerzielle Aspekte wie Verträge \& Profitabilität, wie auch neue Offerings.

Probst, Roger Andreas, Berufsbegleitendes Bachelorstudium in Betriebsökonomie an der Berner Fachhochschule mit Vertiefung in „Management in Practice: Strategy“. Seit mehreren Jahren bei der Berner Bildungszentrum Pflege AG in verschiedenen Funktionen tätig. Aktuell im Fachbereich Weiterbildung als Sachbearbeiter diverser Weiterbildungsangebote von A bis Z sowie als Schnittstellenfunktion zwischen internen und externen Kundengruppen inklusive Mitarbeit in Teilprojekten.

Kim Oliver Tokarski ist Dozent für Innovation, Entrepreneurship, Strategie, Unternehmensentwicklung, Organisation \& Leadership. Er ist Leiter der Abteilung Weiterbildung am Departement Wirtschaft der Berner Fachhochschule sowie in dieser Funktion Mitglied der Departementsleitung. Weiterhin ist er Studiengangsleiter unterschiedlicher Weiterbildungsprogramme. Kim Oliver Tokarski war lange Jahre Gastprofessor an der Wirtschaftsuniversität Bukarest (ASE Bukarest), Rumänien. Seine Forschungs-, Dienstleistungs- und Lehrtätigkeiten sowie Publikationen liegen in den genannten Themenkontexten.

Open Access Dieses Kapitel wird unter der Creative Commons Namensnennung 4.0 International Lizenz (http://creativecommons.org/licenses/by/4.0/deed.de) veröffentlicht, welche die Nutzung, Vervielfältigung, Bearbeitung, Verbreitung und Wiedergabe in jeglichem Medium und Format erlaubt, sofern Sie den/die ursprünglichen Autor(en) und die Quelle ordnungsgemäß nennen, einen Link zur Creative Commons Lizenz beifügen und angeben, ob Änderungen vorgenommen wurden.

Die in diesem Kapitel enthaltenen Bilder und sonstiges Drittmaterial unterliegen ebenfalls der genannten Creative Commons Lizenz, sofern sich aus der Abbildungslegende nichts anderes ergibt. Sofern das betreffende Material nicht unter der genannten Creative Commons Lizenz steht und die betreffende Handlung nicht nach gesetzlichen Vorschriften erlaubt ist, ist für die oben aufgeführten Weiterverwendungen des Materials die Einwilligung des jeweiligen Rechteinhabers einzuholen.

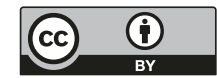

